ARTICLE

\title{
A nanobody-based fluorescent reporter reveals human $\alpha$-synuclein in the cell cytosol
}

\author{
Christoph Gerdes (10 1, Natalia Waal (10 2, Thomas Offner ${ }^{3,4}$, Eugenio F. Fornasiero (1) 1, Nora Wender ${ }^{1}$, \\ Hannes Verbarg1, Ivan Manzini (10 3,4, Claudia Trenkwalder 5,6, Brit Mollenhauer 6,7, Timo Strohäker ${ }^{8}$, \\ Markus Zweckstetter ${ }^{8,9}$, Stefan Becker (10 9 , Silvio O. Rizzoli,10, Fitnat Buket Basmanav 1,11,12 \& \\ Felipe Opazo (1) 1,2,12凶
}

\begin{abstract}
Aggregation and spreading of $\alpha$-Synuclein ( $\alpha$ Syn) are hallmarks of several neurodegenerative diseases, thus monitoring human $\alpha \operatorname{Syn}(h \alpha S y n)$ in animal models or cell cultures is vital for the field. However, the detection of native haSyn in such systems is challenging. We show that the nanobody NbSyn87, previously-described to bind haSyn, also shows cross-reactivity for the proteasomal subunit Rpn10. As such, when the NbSyn87 is expressed in the absence of haSyn, it is continuously degraded by the proteasome, while it is stabilized when it binds to haSyn. Here, we exploit this feature to design a new Fluorescent Reporter for haSyn (FluoReSyn) by fusing NbSyn87 to fluorescent proteins, which results in fluorescence signal fluctuations depending on the presence and amounts of intracellular haSyn. We characterize this biosensor in cells and tissues to finally reveal the presence of transmittable $\alpha$ Syn in human cerebrospinal fluid, demonstrating the potential of FluoReSyn for clinical research and diagnostics.
\end{abstract}

\footnotetext{
${ }^{1}$ Department of Neuro- and Sensory Physiology, University Medical Center Göttingen, D-37073 Göttingen, Germany. ${ }^{2}$ Center for Biostructural Imaging of Neurodegeneration (BIN), University Medical Center Göttingen, D-37073 Göttingen, Germany. ${ }^{3}$ Institute of Animal Physiology, Department of Animal Physiology and Molecular Biomedicine, Justus-Liebig University Giessen, 35390 Giessen, Germany. ${ }^{4}$ Institute of Neurophysiology and Cellular Biophysics, University of Göttingen, Göttingen, Germany. ${ }^{5}$ Department of Neurosurgery, University Medical Center Göttingen, D-37075 Göttingen, Germany. ${ }^{6}$ Paracelsus-Elena-Klinik, Klinikstraße 16, 34128 Kassel, Germany. ${ }^{7}$ Department of Neurology, University Medical Center Göttingen, D-37075 Göttingen, Germany. ${ }^{8}$ German Center for Neurodegenerative Diseases (DZNE), Von-Siebold-Str. 3a, 37075 Göttingen, Germany. ${ }^{9}$ Department for NMRbased Structural Biology, Max Planck Institute for Biophysical Chemistry, 37077 Göttingen, Germany. ${ }^{10}$ Cluster of Excellence "Multiscale Bioimaging: from Molecular Machines to Networks of Excitable Cells" (MBExC), University of Goettingen, Göttingen, Germany. ${ }^{11}$ Campus Laboratory for Advanced Imaging, Microscopy and Spectroscopy, University of Göttingen, D-37073 Göttingen, Germany. ${ }^{12}$ These authors contributed equally: Fitnat Buket Basmanav, Felipe Opazo. ${ }_{\text {email: fopazo@gwdg.de }}$
} 
-synuclein (aSyn) aggregation disorders including Parkinson's disease (PD), Lewy Body dementia, and multiple system arophy, are a group of disorders characterized by the pathological occurrence of intracellular inclusions filled with insoluble aggregates of aSyn. These aggregations can be found in various cell types and regions of the central nervous system ${ }^{1}$. aSyn is a 140 amino-acid long protein that is highly enriched in presynaptic nerve terminals ${ }^{2}$. Despite extensive efforts, the central molecular and physiological role of aSyn remains to be determined. Emerging evidence, however, suggests aSyn to be involved in the regulation and possible maturation of synaptic vesicles ${ }^{3}$, particularly via its role in the assembly of N-ethylmaleimidesensitive factor attachment receptor complexes ${ }^{4}$; key players in synaptic vesicle docking and fusion with the presynaptic membrane. In addition, other functions have been attributed to aSyn including the regulation of glucose levels ${ }^{5}$, serving as an antioxidant $^{6}$ or a chaperone ${ }^{7}$, and suppressing apoptosis in dopaminergic neurons 8 .

Under normal physiological conditions, aSyn is found as a monomeric protein existing in equilibrium between an intrinsically disordered form in the cytosol and a membrane bound, $\alpha$ helical form ${ }^{9}$. Although still subjected to debate ${ }^{10}$, observation of helical $\alpha$ Syn tetramers has also been reported under certain native environments ${ }^{11}$.

It is widely accepted that arrangements of aSyn monomers into small-to-intermediate oligomeric or larger insoluble assemblies ${ }^{12}$ is associated with pathogenesis of aSyn aggregation disorders ${ }^{13}$. Importantly, there is growing evidence for prion-like cell-to-cell transmission properties of different aSyn conformations where "toxic" aSyn species with seeding properties are suggested to get internalized by a host cell and trigger the aggregation of endogenous aSyn ${ }^{14-18}$. There are nevertheless several assumptions, unknown molecular steps and contradictory observations that render the toxic transmission of human aSyn (haSyn) an ambiguous notion. These mainly concern pinpointing of the disease underlying aSyn species (e.g., oligomers vs fibrils, phosphorylated aSyn, etc) ${ }^{19}$, mechanistic steps of the cell-to-cell transmission paradigm 10,20 and the downstream effects of pathological aSyn accumulation that eventually lead to neuronal injury 21 .

Development of tools that can be used for reliable and reproducible detection and tracking of aSyn in in vitro or in vivo is a very important goal for deciphering molecular mechanisms of disease pathogenesis. A common strategy employed in studies addressing the cellular uptake, seeding, and transmission phenomena involve manipulation of the aSyn protein itself. Accordingly, aSyn is either pre-labeled with fluorescent dyes ${ }^{15,22,23}$, or recombinantly expressed as a fusion protein. Concerning the latter, several different approaches are described which include tagging aSyn with a small epitope tag (e.g., myc, V5, etc.) for subsequent antibody-mediated immunodetection ${ }^{15,24}$, expressing it as a fluorescent fusion protein (e.g., YFP- $\alpha$ Syn, DsRed- $\alpha$ Syn, etc.) for direct visualization 25,26 or employing the protein complementation assay principles whereby aSyn is tagged with either $\mathrm{N}$-terminal or C-terminal portions of a split fluorescent or bioluminescent reporter ${ }^{27}$. Although they have proven to be very helpful in advancing the knowledge about synuclein-related pathologies, one common caveat in such approaches is their limited potential in recapitulating the natural behavior of untagged native aSyn. For example, it has been shown that fluorescent protein fusions might result in the wrong localization of the studied protein ${ }^{28}$ or some organic dyes have a propensity to bind to biological membranes ${ }^{29}$. Considering that a critical role is attributed to the lipid interacting properties of aSyn in its pathological behavior ${ }^{30}$, the employment of untagged or native forms of aSyn may be a more-appropriate strategy when attempting to investigate the molecular mechanisms of aSyn pathology. The detection of untagged, native, or endogenous aSyn in in vitro or in vivo model systems requires the use of other tools. Certain dyes such as Thioflavin S, derived from the histological dye Congo red, have been commonly utilized for detecting mature protein aggregates in in vivo models of aSyn propagation and PD pathology 16,31 . However, these dyes have the disadvantage of binding any protein capable of taking an amyloid conformation and thus do not provide an exclusive labeling of $a \mathrm{Syn}^{32}$. In contrast, antibody-mediated immunodetection is a conventional approach for specific detection of aSyn. Hereby, a large number of aSyn-targeting antibodies, including conformation specific-ones and engineered antibody fragments are commonly utilized in a variety of applications ${ }^{33,34}$. In the recent years, camelid-originated single-domain antibodies, also termed nanobodies, emerged as a promising alternative as they confer several advantages including recombinant production, enhanced tissue penetration, small size (ideal for super-resolution microscopy), and the ability to be expressed as intrabodies in mammalian cells ${ }^{35-37}$. The latter feature is particularly attractive as it confers the ability to track and manipulate specific target proteins in living cells ${ }^{38}$. Nanobodies are increasingly being used for investigation of diseases associated with protein misfolding and aggregation. Recently, two nanobodies against aSyn have been identified, NbSyn2 (ref. ${ }^{39}$ ) and NbSyn87 (ref. ${ }^{40}$ ), each binding distinct epitopes at the C-terminal region of aSyn. These have been biochemically well characterized and assessed for their potential therapeutic use by several studies $^{39-44}$.

In this study, we make use of a previously unknown feature of NbSyn87, namely its weak affinity to the $26 \mathrm{~S}$ proteasomal subunit protein Rpn10, which is located at the entrance of the proteasome $\mathrm{e}^{45}$ and functions as a receptor for poly-ubiquitinated proteins that will undergo proteolysis. We show that this interaction is sufficient to drive a continuous proteasome-mediated degradation of intracellularly expressed NbSyn87 unless it is bound to haSyn. The presence of haSyn, on the other hand, results in the avoidance of the degradation of NbSyn 87 by formation of a stabilized NbSyn87:haSyn complex. Accordingly, we exploit this mechanism to create and characterize a nanobodybased Fluorescent Reporter for human aSyn (FluoReSyn), which is able to report the presence or absence of cytosolic haSyn. Our results demonstrate the unique ability of FluoReSyn to report small amounts of cytosolic haSyn in cell lines and transduced primary rat hippocampal neurons. Expression of FluoReSyn in olfactory system of Xenopus laevis also shows its ability to operate and report haSyn in vivo. Furthermore, cells stably expressing FluoReSyn (Reporter-cells) report the presence of haSyn in their cytoplasm after exposing them to human cerebrospinal fluid (CSF) samples. The results presented here indicate that this biosensor is a valuable instrument for studying the transmission of aSyn and has great potential to be further optimized and validated as a diagnostic tool for aSyn aggregation disorders.

\section{Results}

Reporting the presence of untagged haSyn in the cytoplasm. We had previously observed in cells transiently expressing the NbSyn87 (ref. ${ }^{40}$ ) fused to EGFP that their fluorescent signal correlated with the presence or absence of haSyn (Fig. 1a). In an attempt to comprehend this observation, we used the Basic Local Alignment Research Tool (BLAST) to find out if the described haSyn epitope sequence (VDPDNEAYEMPS) ${ }^{40}$ that is recognized by the NbSyn 87 might be present in another endogenous protein. The BLAST result showed a high \% identity (Fig. 1b) to a subunit of the $26 \mathrm{~S}$ proteasome (the $26 \mathrm{~S}$ proteasome non-ATPase regulatory subunit four homolog, also known as Rpn10). This 
a

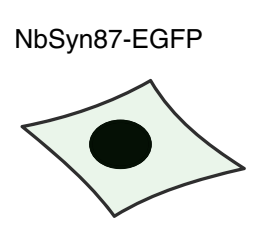

b

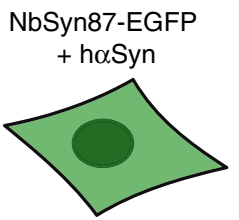

NbSyn 87

putative epitope
hoSyn (NP 000336)

Human Rpnīo (NP_002801.1)

Rat Rpn10 (NP 1'12621.1)

Mouse Rpn10 (NP 00126

Xenopus Rpn10 (NP_001084296.1) 336 SVLENLPGVDPNNEAIRNAMGSLASQASKDN

C

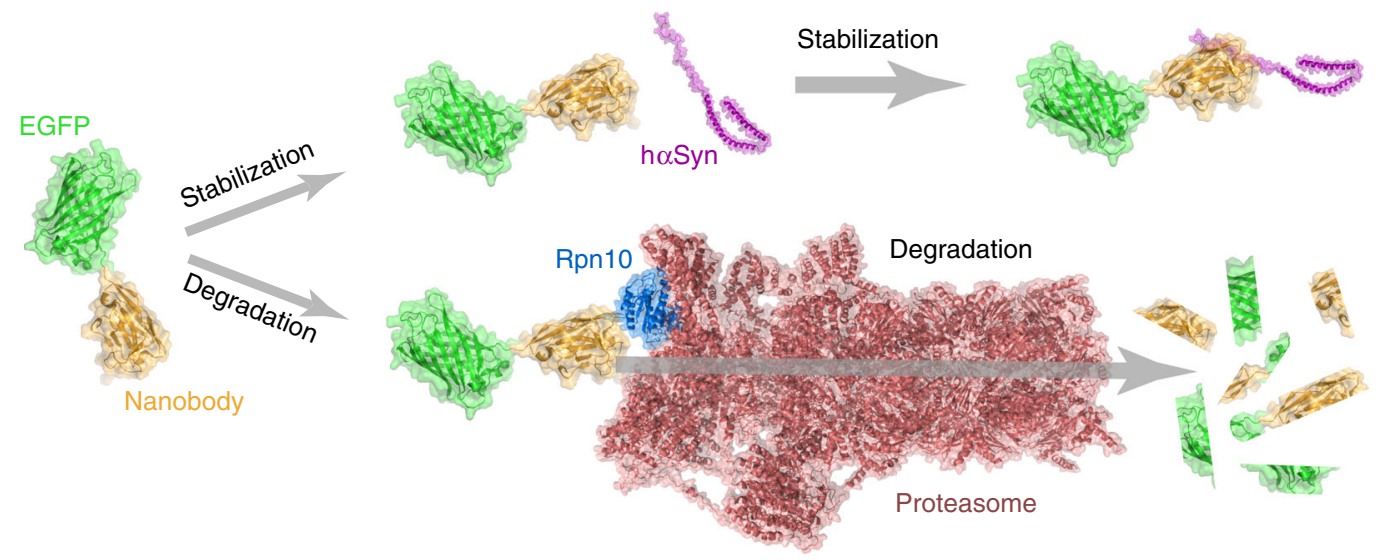

d

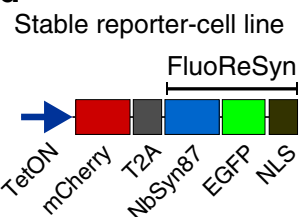

Transient transfection

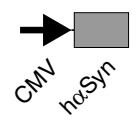

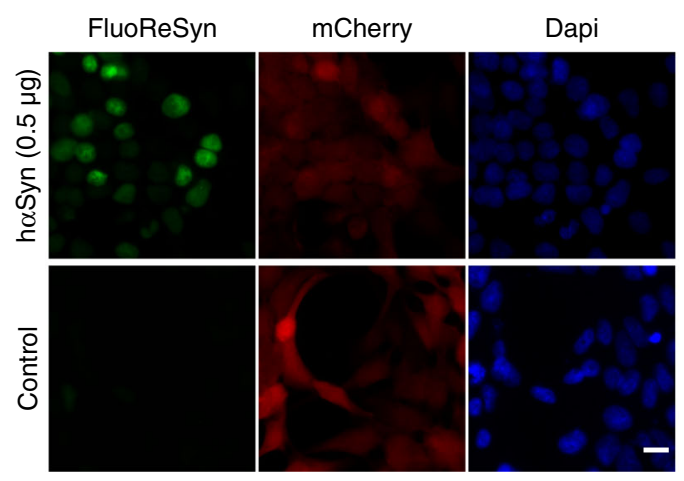

e

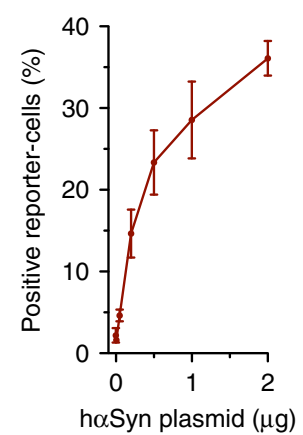

f

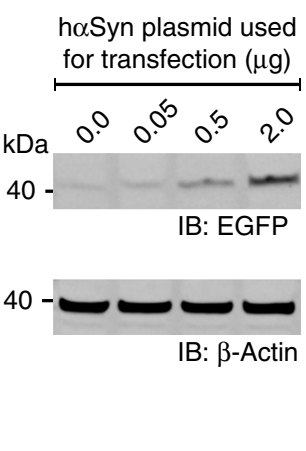

Fig. $1 \alpha$ Syn-dependent accumulation of FluoReSyn in HEK293 cells. a Schematic representation of the initial observation of cells transiently expressing NbSyn87-EGFP alone (left) or together with hoSyn (right). Although the former group of cells showed minimal fluorescence, the latter presented with a strong EGFP signal. b Alignment of the amino-acid sequence from hoSyn and Rpn10 across different species. Rpn10 residues similar to haSyn in the putative epitope are displayed in red. c Schematic representation of the proposed mechanism of degradation versus stabilization of SynNb87-EGFP in the presence or absence of hoSyn. The following Protein Data Bank (PDB) accession numbers were used and modified to assemble the schematic: 2 YOG (EGFP), $2 \times 6 \mathrm{M}$ (Nanobody), 1XQ8 (haSyn), 6MSK (Proteasome). d Schemes of constructs used to transfect HEK293 cells (left). For the stably transfected cells, a tetracycline-inducible promotor (TetON) was used, followed by a mCherry reporter sequence, a cleavable T2A sequence, and FluoReSyn made of NbSyn87, EGFP and a nuclear localization signal (NLS) sequence. Transient expression of untagged wild-type human haSyn was driven by a plasmid containing a cytomegalovirus (CMV) promotor. Equally scaled, representative images of doxycycline-induced Reporter-cells (right). Cells were either mock transfected (control) or transiently transfected with the hoSyn expression constructs. Scale bar represents $10 \mu \mathrm{m}$ e Quantitative analysis of EGFP-positive cells transfected with variable quantities of hoSyn plasmid. Per replication and condition $>1000$ cells were analyzed. Error bars represent the SEM from three independent experiments $(n=3)$. $\mathbf{f}$ Western blot analysis of lysates from Reporter-cells transfected with variable quantities of hoSyn. Immunoblotted (IB) anti-EGFP represents FluoReSyn. Loading control is IB Beta-Actin. Full length blots are displayed in Supplementary Fig. 1d. Source data is available as a Source data file.

protein resides at the entrance of the $26 \mathrm{~S}$ proteasome ${ }^{45}$ and has an important role in the recognition of poly-ubiquitinated proteins that will be processed in the ubiquitin proteasome-mediated proteolysis (UPP) ${ }^{46}$. Using a dot-blot assay with purified Rpn10 and haSyn, we were able to verify that the NbSyn 87 can bind weakly to human Rpn10 (Supplementary Fig. 1a, b). Therefore, taking our results together, we hypothesized that the degradation of NbSyn 87 in the absence of haSyn may be mediated by its weak but continuous recruitment to the proteasome upon binding the endogenous Rpn10 (Fig. 1c).

In order to test our hypothesis and further characterize NbSyn87 in terms of this special feature, we decided to generate a stable cell line expressing NbSyn87 fused to EGFP and have
mCherry signal as an expression reporter using the self-cleavable domain $\mathrm{T}_{2} \mathrm{~A}^{47}$ (Fig. 1d). In addition, we added a NLS sequence at the C-terminus of NbSyn87-EGFP to concentrate the EGFP signal in the nucleus and gain sensitivity during imaging. The expression of this protein-chimera was controlled under the tetracycline-inducible promotor system (TetOn). Optimal induction duration to maximize the NbSyn87-EGFP-NLS expression was determined by analyzing the mCherry reporter signal (Supplementary Fig. 1c). In line with our original observations, we detected a clear nuclear EGFP signal in doxycycline-induced NbSyn87-EGFP-NLS stable cell line when we transiently transfected them with wild type and untagged haSyn (Fig. 1d). The strength of this effect was dependent on the amount of haSyn 
a

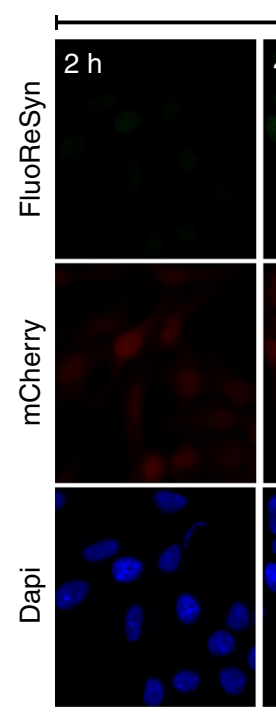

$1 \mu \mathrm{M}$ MG132

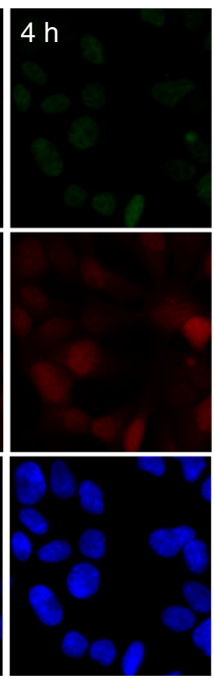

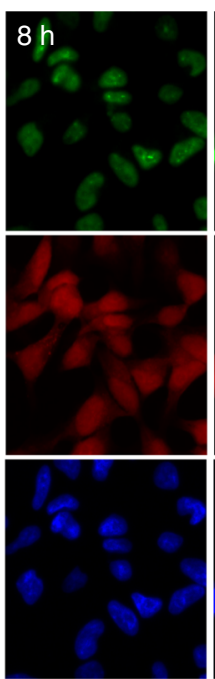

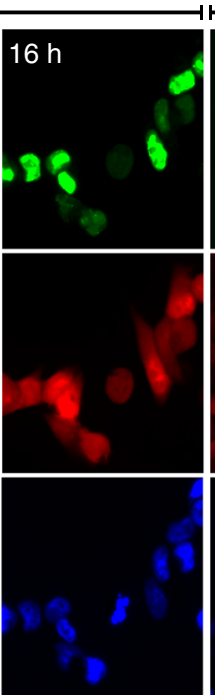

No MG132

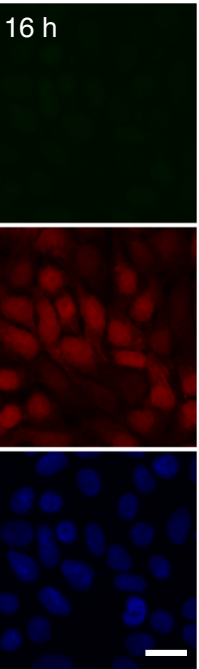

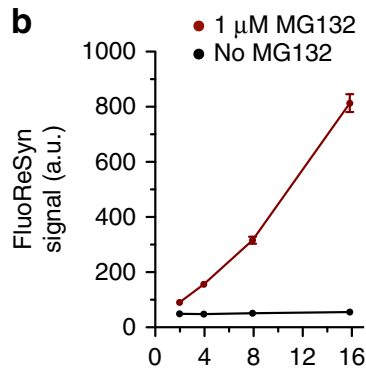

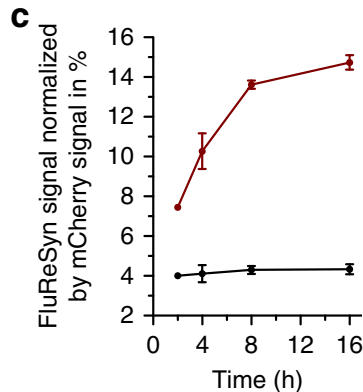

Fig. 2 The FluoReSyn is rapidly degraded by the proteasome machinery. a Equally scaled, representative epifluorescence images of MG132 treated and untreated Reporter-cells which were fixed 2, 4, 8, or $16 \mathrm{~h}$ post treatment. The FluoReSyn accumulates in response to prolonged MG132 treatment of Reporter-cells as revealed by the increasing nuclear EGFP intensity. Reporter-cells untreated with MG132 yielded virtually no EGFP signal even after $16 \mathrm{~h}$. Scale bar represents $20 \mu \mathrm{m}$. b Quantitative analyses of FluoReSyn signal (EGFP) in arbitrary units (a.u.) when Reporter-cells were treated or untreated with MG132. Per replication and condition more than 540 cells were analyzed. c FluoReSyn signal normalized by mCherry signal (EGFP to mCherry signal intensity). Error bars represent the SEM from three independent experiments $(n=3)$. Source data is available as a Source data file.

present in the cells; detected both by EGFP fluorescence signal intensity analysis (Fig. 1e) and EGFP amounts revealed in western blots (Fig. If and Supplementary Fig. 1d). Doxycycline-induced cells that were not transiently transfected with haSyn displayed the mCherry reporter signal but no EGFP signal (Fig. 1d). Therefore, we termed the NbSyn87-EGFP-NLS construct as the Fluorescent Reporter for aSyn (FluoReSyn) and the stably FluoReSyn expressing cell line as the Reporter-cells.

Proteasome-mediated degradation of FluoReSyn. In order to validate our proposed mechanism for the degradation of FluoReSyn, we treated doxycycline-induced Reporter-cells (without expressing haSyn) over different time periods with the MG132 proteasome inhibitor. MG132 concentration was optimized to provide a strong gain of EGFP and mCherry signal while minimizing its adverse toxic effects on the Reporter-cells (Supplementary Fig. 2). The results clearly showed that, following treatment with $1 \mu \mathrm{M}$ of MG132, FluoReSyn started to accumulate in the nuclei of Reporter-cells already from the 4 th $\mathrm{h}$ on without the presence of haSyn (Fig. 2a, b). Induced Reporter-cells untreated with MG132, showed in contrast virtually no FluoReSyn signal throughout the whole duration (Fig. 2a, b). These suggested that the reporter is regularly produced but also continuously degraded via the proteasome in the cell (fast turnover) under normal conditions. The mCherry and FluoReSyn are produced from a single mRNA, making a fusion protein that is efficiently cleaved at the T2A domain ${ }^{47}$. It is expected that this strategy results in stoichiometric amounts of FluoReSyn and mCherry, which allowed us to normalize the signal of FluoReSyn with the mCherry signal. In induced Reporter-cells untreated with MG132, the relation of FluoReSyn to mCherry signal was maintained during the $16 \mathrm{~h}$ experiments (Fig. 2c). However, when using MG132, already after $2 \mathrm{~h}$, the ratio between FluoReSyn signal and mCherry doubled and kept growing over time, showing that the accumulation of FluoReSyn exceeds that of mCherry and that the former gets particularly enriched in the
Reporter-cells when the proteasome machinery is inhibited (Fig. 2c). This observation suggests that the proteasomal degradation is particularly accelerated for FluoReSyn under normal conditions (uninhibited proteasome machinery), and thus substantiates our proposition that the nanobody NbSyn 87 specifically targets the proteasome and is degraded by it.

FluoReSyn reports haSyn in vivo. As Rpn10 is a well-conserved protein across different species (Fig. 1b), we presumed that our proposed mechanism should also operate in a model organism that lacks endogenous haSyn and possesses the conserved Rpn10 epitope recognized by NbSyn87. Accordingly, we chose to validate the proposed mechanism in living Xenopus laevis tadpoles, a time- and cost-efficient model organism ${ }^{48}$. Xenopus laevis expresses endogenously the same Rpn10 epitope needed for FluoReSyn to operate and offers a straightforward electroporation-mediated approach for gene delivery to the olfactory receptor neurons in the olfactory epithelium of living animals $^{49}$ (Fig. 3a, b). Accordingly, the plasmid encoding for FluoReSyn was electroporated either alone or together with a plasmid encoding for haSyn fused to mCherry into the right or left nostrils of anesthetized tadpoles, respectively. By in vivo imaging of tadpoles with two-photon microscopy we observed many GFP-positive nuclei co-localizing with the mCherry signal (hASyn) in the left nostrils of the animals (Fig. 3c, e, g, high magnification example on $3 \mathrm{j}$ ), which was clearly in contrast with the right nostrils presenting seldom any GFP-positive nucleus (Fig. 3d, f, h). Analysis of the distribution of GFP fluorescence intensity further confirmed the clear distinction between left and right nostrils (Fig. 3i). Altogether, these data further validated our previous conclusions by demonstrating that the same mechanism seems to be operational in vivo whereby FluoReSyn is stabilized upon haSyn binding and cleared from the cell by proteasome-mediated degradation in the absence of this interaction. 

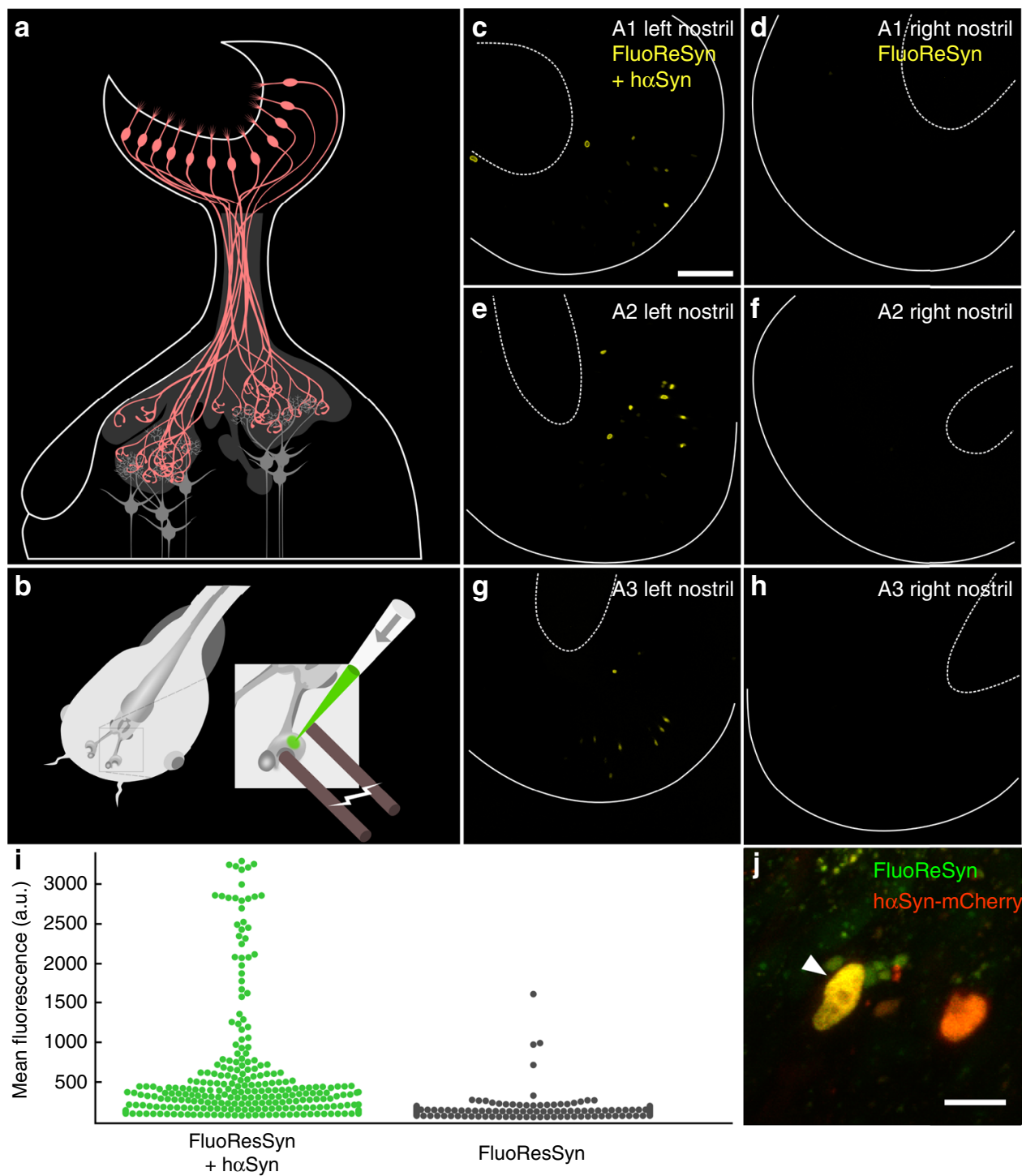

FluoResSyn

Fig. 3 in vivo FluoReSyn activity in neurons of larval Xenopus laevis. a Organization of the Xenopus laevis olfactory system: olfactory receptor neurons (ORNs; red) residing in the olfactory epithelium project their axons to the olfactory bulb, where they synapse onto the second order projection neurons (gray). b Schematic of plasmid electroporation into the olfactory mucosa of anaesthetized Xenopus laevis tadpoles. $\mathbf{c}, \mathbf{e}, \mathbf{g}$ Left nostrils of three animals (A1-A3) were co-electroporated with FluoReSyn- and haSyn-expressing plasmid. Scale bar represents $100 \mu \mathrm{m}$. $\mathbf{d}, \mathbf{f}, \mathbf{h}$ Respective right nostrils electroporated only with FluoReSyn. For all animals and nostrils identical plasmid concentrations and imaging settings were used. Basal and apical delineations of olfactory epithelium are indicated by line and dashed line, respectively. $\mathbf{i}$ Distribution of FluoReSyn mean fluorescence intensity of positive nuclei from the left nostrils (FluoReSyn and hoSyn plasmid; green dots) and nuclei from the right nostrils (FluoReSyn plasmid only; black dots) in arbitrary units (a.u.). More than 500 nuclei were analyzed from nine animals $(n=9)$ imaged in vivo. Unpaired and two tailed student $t$ test results in $p<0.0001$ $\left({ }^{\star \star \star \star}\right)$. $\mathbf{j}$ High magnification example of a neuron co-expressing FluoReSyn (green) and hoSyn-mCherry (red), resulting in nuclear colocalization (yellow) of both proteins. Scale bar represents $10 \mu \mathrm{m}$. Source data is available as a Source data file.

The specificity of the FluoReSyn for haSyn. As a next step, we assessed whether FluoReSyn binds specifically to human aSyn or has an affinity towards other synuclein species. For this purpose, Reporter-cells were transiently transfected with plasmids encoding for haSyn, human ßSyn (hßSyn), or rat aSyn (raSyn). We analyzed the proportion of cells with a positive EGFP signal by epifluorescence microscopy (Fig. 4). The expressions of the different synuclein species were controlled by immunostaining with an antibody that recognizes all transfected variants. Although no significant differences were observed among the different synuclein species in terms of their expression (Supplementary Fig. 3a), only in haSyn-transfected Reporter-cells a substantial proportion of the cells were positive for the FluoReSyn signal (Fig. 4b). This result, therefore, proposes that FluoReSyn binds primarily to human $\alpha$ Syn. It is important to note that the epitope sequence of the haSyn recognized by NbSny 87 has a great identity with the raSyn sequence with the epitopes differing from each other only by two residues (Supplementary Fig. 3b). It is noteworthy, that FluoReSyn seems to bind with higher affinity to Rpn10 than to the raSyn.

Detection of haSyn uptake from the culture medium. In relation to the pathological aSyn transmission phenomenon ${ }^{10}$, we evaluated the ability of FluoReSyn to report the entry of foreign haSyn into the cellular cytosol. For this purpose, purified recombinant haSyn was administered to the culture mediums of induced Reporter-cells either on its own or in a mixed state with a 

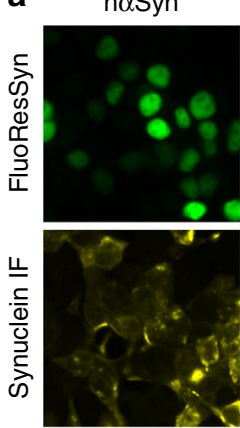

$\frac{3}{0}$

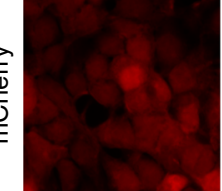

hßSyn

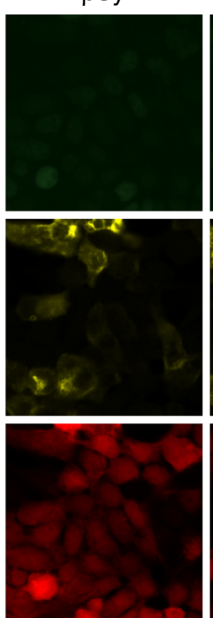

roSyn

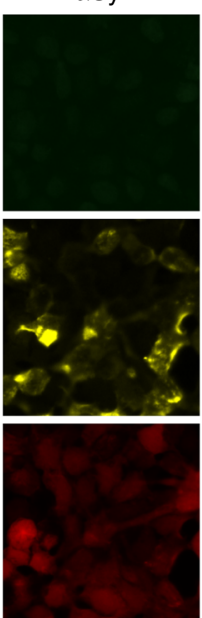

Control

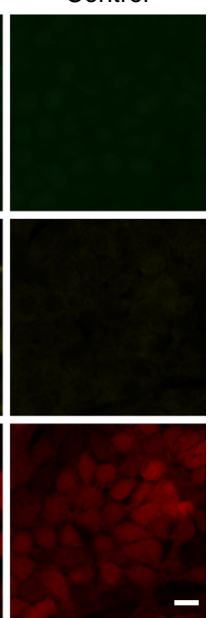

b

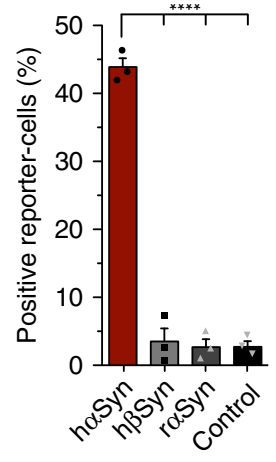

Fig. 4 The FluoReSyn is a specific sensor for human $\boldsymbol{\alpha}$ Syn. a Equally scaled representative epifluorescence images of Reporter-cells transfected with h $\alpha$ Syn, h $\beta$ Syn, or $r \alpha$ Syn expression constructs. Scale bar represents $10 \mu \mathrm{m}$. b Quantification of the proportion of EGFP-positive cells. Error bars represent the SEM from the independent experiments $(n=3)$. Per replication and condition $>6000$ cells were analyzed. Ordinary one-way ANOVA resulted in ${ }^{\star \star \star *} p<0.0001$. Source data is available as a Source data file.

a

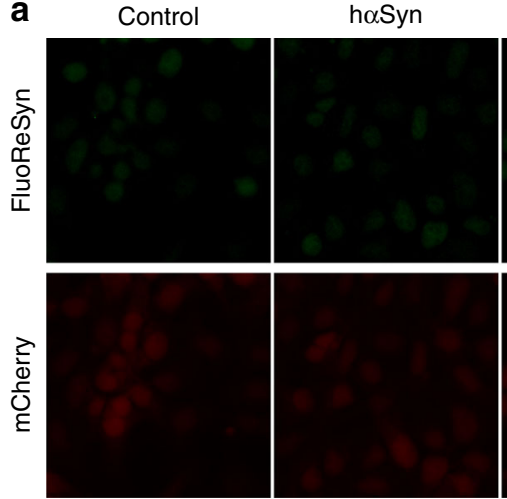

hßSyn + iMAX

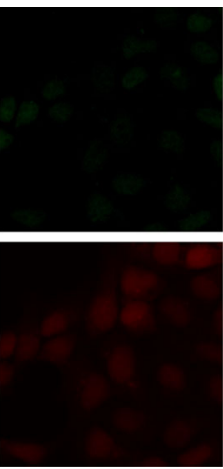

haSyn + iMAX

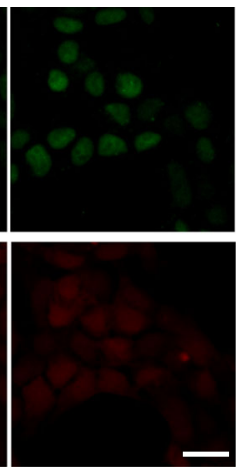

b

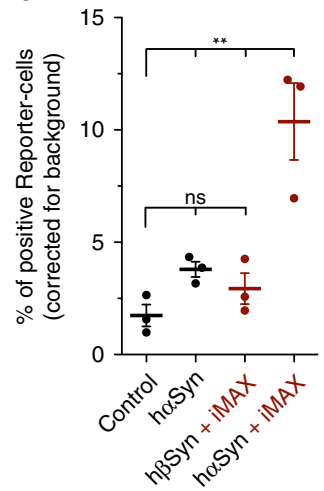

Fig. 5 Transmission of recombinant hoSyn into Reporter-cells. a Equally scaled representative epifluorescence images of Reporter-cells after incubation for $14 \mathrm{~h}$ with recombinant h $\alpha$ Syn monomers added to the culture medium. Monomers were either administered alone (h $\alpha$ Syn) or pre-mixed with the

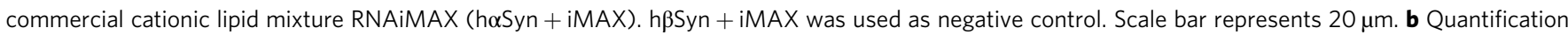
of the percentage of EGFP-positive Reporter-cells. Error bars represent the SEM from three independent experiments $(n=3)$. Per replication and condition $>2300$ cells were analyzed. One-way ANOVA with multiple comparison Tukey's post hoc test ${ }^{\star \star} p<0.01$, ns: not significant. Source data is available as a Source data file.

cationic liposome reagent (i.e., RNAiMAX) to enhance the protein uptake as suggested previously ${ }^{50,51}$. We observed that, if haSyn was associated with RNAiMAX, it manages to get into the cytosol and stabilize FluoReSyn as evidenced by the accumulated EGFP signal in the nucleus (Fig. 5). On the other hand, administrating hßSyn associated with RNAiMAX or just naked haSyn generated only background levels (i.e., induced Reporter-cells not exposed to anything) of EGFP-positive cells, which suggests that haSyn on its own failed to go across the cell plasma membrane efficiently.

Subsequently, we setup a co-culture of Reporter-cells and HEK293 cells stably expressing untagged and wild-type haSyn under an inducible promotor (TetON-haSyn cells; Supplementary Fig. 4) to investigate if mammalian produced haSyn was able to leave the cells and then enter into the neighboring Reportercells. As a control condition, Reporter-cells were co-cultured with wild-type HEK293 cells, which lack endogenous haSyn expression. The analysis of co-cultures maintained for 2-5 days revealed no significant differences between the test and control groups failing to confirm the occurrence of any haSyn transmission events between the haSyn producing cells and the Reporter-cells (Supplementary Fig. 5).

Uptake of haSyn from the culture medium by primary neurons. As a next step, we assessed the functionality of FluoReSyn in neurons, by investigating whether it can report the cytosolic presence of haSyn, after adding recombinant haSyn to the medium of primary neuronal cultures. For this purpose, we prepared rat hippocampal neuron cultures and infected them at DIV 14 with an adeno-associated virus (AAV) encoding for NbSyn87-mCherry-NLS (a red version of FluoReSyn). We administered both the monomeric and large fibrillar ${ }^{52}$ forms of haSyn extracellularly to the culture medium of FluoReSyntransduced hippocampal neurons. The analysis suggested that naked monomeric haSyn can reach the cytosol of neurons and can produce a detectable FluoReSyn signal in their nuclei 
a

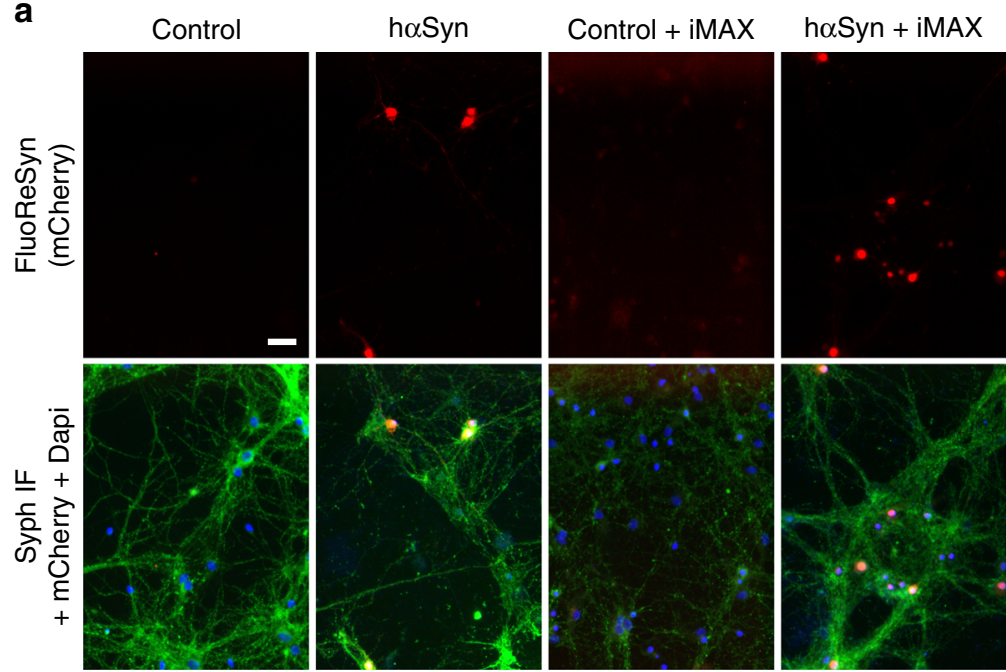

b

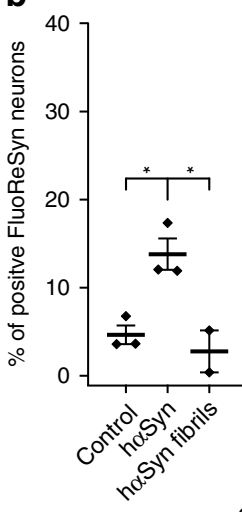

C

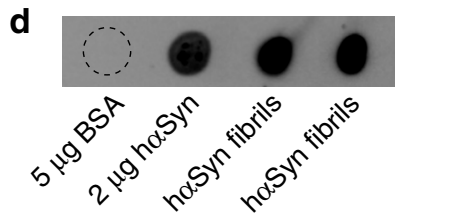

Fig. 6 Detection of hoSyn monomers added to the medium of cultured neurons. a Equally scaled, representative images of primary rat hippocampal neurons infected with adeno-associated virus (AAV) encoding for NbSyn87-mCherry-NLS (a red version of the FluoReSyn). The cultures were then exposed to $20 \mu \mathrm{M}$ of monomeric hoSyn, with or without RNAiMAX (iMAX), for $14 \mathrm{~h}$. Immunofluorescence against synaptophysin was used as a neuronal marker (Syph IF, green signal). The negative controls were neurons receiving Opti-MEM with or without RNAiMAX. Scale bar represents $20 \mu \mathrm{m}$. b, c Quantification of FluoReSyn signal-positive neurons. One-way ANOVA with multiple comparison Tukey test ${ }^{*} p<0.05$. Error bars represent the SEM, each point in the scatter plot represents the average of an independent experiment $\mathbf{d}$ Monomeric untagged hoSyn and two different batches of in vitro produced h $\alpha$ Syn fibrils were spotted on a nitrocellulose membrane and were detected by NbSyn87-Alexa647, which confirmed the ability of NbSyn87 to bind the fibrils used in hippocampal cultures. Bovine serum albumin (BSA) was used as negative control. Source data is available as a Source data file.

(Fig. 6a, b). This was in contrast to the large haSyn fibrils, which failed to induce FluoReSyn signals above that of the control neurons (Fig. 6b).

Based on our previous observations with the Reporter-cell line, where we also administered the synuclein species premixed with the cationic lipid RNAiMAX (Fig. 6a, c). The results revealed a higher proportion of neurons with FluoReSyn positive nuclei when haSyn monomers were pre-mixed with the cationic lipids from RNAiMAX and added to the culture medium (Fig. 6a, c). It was interesting to observe that both, the naked monomers (Fig. 6b) and those complexed with lipids (Fig. 6c) were able to go across the neuron plasma membranes. This is clearly distinctive to the HEK293-based Reporter-cells, which displayed internalization of haSyn only when complexed to cationic lipids (Fig. 5b).

The use of cationic lipids did not influence the outcome for fibrils. Similar to the previous observation with uncoated fibrils (Fig. 6b), no FluoReSyn signal was detected in neurons exposed to RNAiMAX-haSyn fibril complexes (Fig. 6c). Importantly, we showed that NbSyn87 can clearly bind to our in vitro generated fibrils (Fig. 6d), which also confirmed previous reports ${ }^{40}$. Thereby, we can exclude that the negative observations were owing to the inability of FluoReSyn to detect our fibrils (Fig. 6d), but are most likely a proof that fibrils have not succeeded in entering the cytoplasm of neurons.

Detection of haSyn in CSF samples. Confident that FluoReSyn can reliably report cytosolic haSyn, we decided to evaluate the ability of the Reporter-cells to detect haSyn species in humanoriginated biological samples. The main rationale here was to explore the potential usability of this cellular system for future diagnostic purposes. Therefore, we exposed induced Reportercells cultured on a 96-well plate to CSF samples from 42 individuals diagnosed with variable neurological disorders that were unrelated to aSyn aggregation disorders. Reporter-cells not exposed to CSF, as well as wild-type HEK293 cells exposed and not exposed to CSF, were used as negative controls. Cells were fixed $24 \mathrm{~h}$ post treatment and dozens of images of randomized and non-overlapping locations were automatically acquired per well (Fig. 7a). Data analysis showed a small but clear trend of positive Reporter-cells that were incubated with human CSF (Fig. 7b). We also correlated the specific clinical diagnosis of each patient and the total aSyn concentrations in their CSF to the Reporter-cells activity, as displayed in Supplementary Fig. 6. As controls, Reporter-cells not exposed to CSF or wild-type HEK293 cells (not producing any FluoReSyn chimera) incubated with or without CSF, all displayed background levels of positive signal (Fig. 7b). This observation reassured that the small percentage of positive Reporter-cells observed upon CSF exposure is a specific and trustworthy response to some forms of haSyn present in human CSF that can reach the cytosol of our model Reportercells. Altogether, this result not only suggests that human CSF may contain a transmittable form of aSyn that is capable of entering into cells but also opens the possibility to optimize this system for generating a unique cell-based diagnostic tool for aSyn aggregation disorders.

\section{Discussion}

Here, we present for the first time a unique feature of the NbSyn87, namely, its natural tendency to bind to the proteasomal subunit Rpn10 that leads to its own degradation and eventual clearance from the cell cytoplasm in the absence of haSyn. This special feature allowed us to develop FluoReSyn, the nanobodybased fluorescent reporter for haSyn, which is capable of detecting the presence or absence of haSyn in the cellular cytoplasm. Furthermore, our Reporter-cells stably expressing FluoReSyn were able to detect a transmittable form of haSyn present in human CSF. 
a

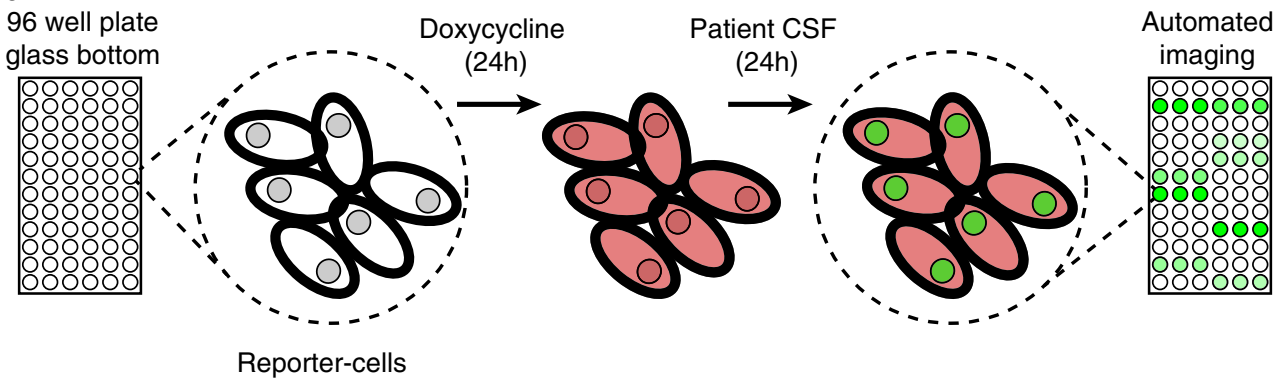

b

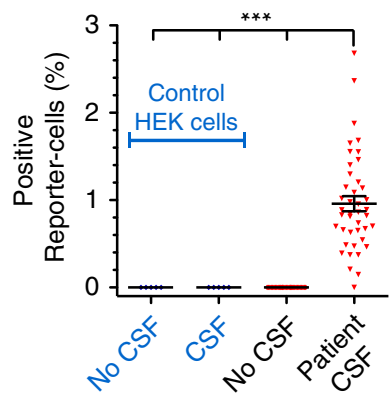

Fig. 7 Detection of transmittable hoSyn from human CSF samples. a Illustration of the experimental setup. Reporter-cells or wild-type HEK293 cells (control) were seeded onto glass bottom 96-well plates. FluoReSyn expression was induced by doxycycline for $24 \mathrm{~h}$ before they were exposed to human CSF or culture medium (control) for another $24 \mathrm{~h}$. Finally, cells were fixed and $\sim 20$ images of random and not overlapping locations were acquired automatically per well. The CSF from each patient was used at least in three different wells. b Quantification of cells with positive FluoReSyn signal. In total, results represent data from 126 wells of Reporter-cells receiving CSF samples from 42 individuals in technical triplicates, 102 negative control wells with Reporter-cells receiving culture medium, 15 wells of HEK293 cells receiving CSF samples from five individuals in technical triplicates and 36 wells of HEK293 cells receiving culture medium. One-way ANOVA with multiple comparison Tukey test ${ }^{\star \star \star} p<0.001$. Further correlations between the Reportercell activity and the clinical information of each patient are displayed in Supplementary Fig. 6 and Supplementary Table 1. Error bars represent the SEM, each data point represents the average from three replicas (wells) of an individual patient's CSF. Source data is available as a Source data file.

Uptake of toxic aSyn species by cells and their subsequent intracellular trafficking is a crucial part of the proposed aSyn transmission pathology ${ }^{20}$. Accordingly, we first characterized and assessed the potential of FluoReSyn Reporter-cells to be used as a research tool for investigating the transmission phenomenon. Our results after introducing recombinant aSyn to the culture medium suggested that the Reporter-cells could reliably report the uptake of extracellular aSyn. Similar to other reports ${ }^{51,53,54 \text {, }}$ we did not detect direct cytoplasmic internalization of naked monomeric haSyn by the Reporter-cells (derived from HEK293 cells). We rather observed that the cytosolic uptake required assistance with lipid-based elements as also had been shown in other studies ${ }^{51,54}$. Different from the HEK-based Reporter-cells, we observed that entry of naked recombinant aSyn monomers into primary neurons expressing FluoReSyn is more likely to occur, and lipid-based facilitating agents like the RNAiMAX make the entry process even more efficient. These observations expose the influence of the cellular context on the translocation of aSyn from the extracellular to the intracellular space (e.g., requirement of distinct receptor interactions or membrane translocators for $\alpha \mathrm{Syn})^{20}$ and can be interpreted as a reflection of the neuronal nature of aSyn transmission pathology. In line with this interpretation, we did not observe a transmission event when HEK293-based cells stably transfected with haSyn were cocultured with the Reporter-cells. On the other hand, these transmission events might be highly dependent on the concentration of haSyn and duration of exposure. With our setup we could not employ higher concentrations of haSyn in order to avoid toxic effects to the cells and furthermore, we were limited in terms of exposure durations as a longer maintenance of dividing cells in culture became difficult after several days.

In the current study, we did not observe a positive FluoReSyn signal in transduced neurons following their exposure to fibrillary aSyn. This was not an unexpected observation since fibrils are large structures (in the $\mu \mathrm{m}$ range) that should not enter the cellular cytosol easily, especially as they are known to not form porelike structures on membranes ${ }^{55}$. Accordingly, it is probable that our observations merely reflected the inability of these bulky structures to penetrate into the neuronal cytosol.

It has been proposed that the uptake of larger arrangements such as oligomers, fibrils, or aggregated aSyn might be mediated by regular endocytosis ${ }^{20,22}$. In this case, FluoReSyn would not detect compartmentalized aSyn in endocytosed vesicles unless
aSyn finds a way to escape into the cytosol. It has recently been shown that aSyn pre-formed fibrils (pffs; $<50 \mathrm{~nm}$ of length ${ }^{56}$ ) that were internalized by cultured primary neurons remained confined to endo-lysosomal compartments up to 7 days, without a major escape from the endocytic pathway ${ }^{57}$. Thus, even small fibrils have difficulties penetrating into the neuronal cytosol.

Finally, these results do not exclude the possibility that other types of large assemblies or fibrils do penetrate into the cell cytosol, and would therefore be detected by the sensor. This issue could be tested more thoroughly in the future. It is known that many types of fibril-like assemblies with different morphologies and structures can be obtained by changing the preparation and incubation conditions (e.g., $\mathrm{pH}$, salinity, temperature, presence of modulators ${ }^{58,59}$. Structural variabilities of recombinant fibrils were also shown to propagate to in vitro and in vivo functional properties of the formed assemblies ${ }^{19,58,59}$. For example, it has been shown that upon reducing the incubation $\mathrm{pH}$, the morphology of formed high-molecular weight aSyn assemblies shifted from fibrillary to more amorphous with the latter showing reduced in vitro seeding efficienc ${ }^{58}$. Another study demonstrated that simply by changing from physiological salt concentrations to a lower salt condition, or by adding a chelating agent to the incubation buffer the appearance of the formed highmolecular weight assemblies of haSyn changed from cylindrical to flat. These assemblies, referred to as fibrils or ribbons, respectively, were shown not only to differ in other structural aspects but also in terms of their in vitro-, in vivo seeding, and propagation properties as well as the degrees of cellular toxicity they induced ${ }^{59}$.

Our results demonstrated that the FluoReSyn works both in a cell line and primary neurons, and can report the uptake of recombinant aSyn monomers. Introducing recombinant or tissue derived aSyn extracellularly to in vitro cell cultures is a common approach for investigating the internalization and subsequent seeding activities of aSyn in the context of transmission paradigm $^{23,60,61}$. However, the readily available cellular models and tools are generally limited in terms of offering an unambiguous and spatially resolved discernment of internalized aSyn from the extracellularly applied aSyn seed ${ }^{57}$. Therefore, there is a necessity to develop novel tools that would enable selective visualization of internalized aSyn and deliver a quantitative characterization of aSyn uptake phenomenon ${ }^{57}$. In a recent study where neuronal cultures were exposed to GFP-tagged aSyn 
pre-formed fibrils (pffs), a membrane-impermeable fluorescence quencher dye was used to exclusively quench the fluorescence of extracellular fibrils, thus enabling the selective imaging of only internalized seeds ${ }^{57}$. Similarly, we propose that, FluoReSyn is an optimal tool for this purpose as it can exclusively detect cytosolic aSyn. A major advantage of our system is that it avoids the necessity of employing tagged or covalently conjugated forms of aSyn, which as discussed previously may not recapitulate the normal behavior of native, untagged aSyn. Accordingly, provided that the nanobody epitope is exposed, FluoReSyn can be used for tracking the behavior of any endogenous species of native aSyn obtained from human materials.

Our data suggest that FluoReSyn is a useful research tool not only for neuronal cultures, but also for in vivo set-ups like in our experiments with living X. Laevis. Besides, the FluoReSyn's specificity to haSyn would be of advantage when studying haSyn transmission in animal models (e.g., mice, rat) as any interference from endogenous aSyn present in rodents can be disregarded.

As a next step in further characterizing the cellular reporter system, we evaluated its ability to detect human-originated aSyn with the future perspective of developing it further into a diagnostic tool ${ }^{62-65}$. Here, we used CSF samples from a readily available cohort of individuals (42). The results showed that our Reporter-cells could indeed detect specific species of aSyn in human CSF, which are able to enter into their cytoplasm. Considering our observations with recombinant aSyn monomers that required a lipid coating agent for entering the Reporter-cells, it is plausible that the aSyn molecules we detected in CSF samples were in vesicular structures such as exosomes as already suggested by others ${ }^{66,67}$, or were species other than monomers, such as oligomers with different proposed means of entering the cells ${ }^{68-71}$.

The presence of aSyn in CSF can be measured biochemically (e.g., ELISA $)^{72}$, and many efforts have been directed at developing assays for detection of CSF-originated $\alpha$ Syn as a biomarker ${ }^{73,74}$. It is important to emphasize that our system provides extra information by detecting haSyn forms that are able to get into the cytosol of cells. Therefore, it is plausible that we are detecting the species that are more prone to transmission related pathology. This is particularly relevant in the light of emerging evidence, which suggests that particular species of aSyn (e.g., oligomers, aggregates) that are associated with toxicity can serve as far-better biomarkers than total $\alpha \mathrm{Syn}$ in $\mathrm{CSF}^{74}$.

Although our initial results in Fig. 1 show that the FluoReSyn signal correlates to the amount of aSyn, our Reporter-cells are not yet quantitative enough to precisely determine the concentrations of transmittable aSyn in human CSF. Nevertheless, this is a fascinating first proof-of-concept. To the best of our knowledge, our cellular haSyn reporter system is so far the sole approach developed for the detection of transmittable haSyn species present in human body fluids. We believe that major optimizations can be performed to this system to increase its sensitivity and accuracy. These enhancements might include employment of a faster maturing and brighter EGFP variant, tuning of the FluoReSyn sensitivity biochemically, and destabilizing the antigen-unbound NbSyn87 (ref. ${ }^{75}$ ). Once these optimizations are performed and validated, a logical next step would be the generation of a knock-in (KI) mice, to thus generate an animal model for studying the mechanical and molecular aspects of aSyn transmission. Using primary cells from these KI mice, it will then be possible to establish a neuron-based reporter system, which would presumably hold more promise in a diagnostic context. We hope such efforts might help to achieve more sensitive and quantitative read-outs, which would in turn pave the way for the development of an accurate and reliable diagnostic or prognostic tool for aSyn associated disorders.

\section{Methods}

Plasmid transfections and virus infections. Transfections with pcDNA $3.1(+)$ vectors (Thermo Fisher Scientific) encoding for haSyn (accession number NM_000345.4), human $\beta$-synuclein (hßSyn; accession number NM_001001502.3) or rat aSyn (raSyn; accession number NM_019169.2) sequences were performed with Lipofectamine 2000 (Invitrogen, Thermo Fisher Scientific) and Opti-MEM I Reduced Serum Medium (Opti-MEM from Gibco, Thermo Fisher Scientific, Waltham, MA, USA) according to manufacturer's instructions. Transfected cells were typically used after $48 \mathrm{~h}$. The AAV coding for NbSyn 87 fused to mCherry and nuclear localization signal (NLS) sequences (NbSyn87-mCherry-NLS) was kindly provided by Dr. Sebastian Kügler, Department of Neurology; Viral Vectors Laboratory, University Medical Center Göttingen, Germany.

Protein purification. Rpn10, haSyn, hßSyn were produced using NEB Express Competent Escherichia coli (New England BioLabs Inc., Ipswich, MA, USA). Bacteria were grown overnight with the plasmid of interest in Lysogeny Broth (Sigma-Aldrich) and the respective antibiotics. Next day, cells were further cultured in Terrific Broth (Sigma-Aldrich) and antibiotics until OD was $\sim 2-3$ at $37^{\circ} \mathrm{C}$ and induced with $0.8 \mathrm{mM}$ IPTG for $4 \mathrm{~h}$. After adding $5 \mathrm{mM}$ EDTA, cells were harvested by centrifugation at $\sim 3000 \times g$ for $30 \mathrm{~min}$ at $10^{\circ} \mathrm{C}$ and frozen at $-20{ }^{\circ} \mathrm{C}$ until further processing. Pellets were resuspended on ice with $1 \mathrm{mM} \mathrm{DTT}, 25 \mathrm{mM}$ Imidazole, and $1 \mathrm{mM}$ PMSF in a His-binding buffer (50 mM HEPES pH 8.0, 500 $\mathrm{mM} \mathrm{NaCl}, 5 \mathrm{mM} \mathrm{MgCl}_{2}$ and $10 \%$ glycerol) and bacteria were lysed by sonication on ice. Cell debris was separated by centrifugation at $\sim 15,000 \times g$ for $1 \mathrm{~h}$ at $4{ }^{\circ} \mathrm{C}$ Clear supernatant was incubated with $2 \mathrm{ml}$ of pre-equilibrated Ni-beads (cOmplete His-Tag Purification Resin, Roche, Switzerland) for $1 \mathrm{~h}$ and then transferred to a sigma column to be washed consecutively with His-binding buffer (50 mM HEPES $\mathrm{pH} 8.0,1000 \mathrm{mM} \mathrm{NaCl}, 10 \mathrm{mM} \mathrm{MgCl}_{2}, 25 \mathrm{mM}$ Imidazole and $5 \%$ glycerol) and low-Salt-pH buffer (50 mM HEPES pH 7.5, $500 \mathrm{mM} \mathrm{NaCl}, 5 \mathrm{mM} \mathrm{MgCl}, 25 \mathrm{mM}$ Imidazole, $5 \%$ glycerol) with each of them containing $25 \mathrm{mM}$ Imidazole. Finally, protein was eluted by $500 \mathrm{mM}$ Imidazole and His-Tag was cleaved with SUMO protease and removed by reverse binding to nickel beads. Pure proteins were desalted into PBS, purity was confirmed by polyacrylamide gels (PAGE) and concentrations were determined using nanodrop spectrophotometer considering the protein molecular weight and extinction coefficient.

NbSyn87 coupling to Alexa647 fluorophore. NbSyn87 protein was obtained from a custom production service offered by NanoTag Biotechnologies $\mathrm{GmbH}$ (Göttingen, Germany), and the nanobody was equipped with one ectopic cysteine at its C-terminal. $1 \mathrm{mg}$ of pure NbSyn 87 was reduced by adding TCEP (SigmaAldrich) to a final concentration of $5 \mathrm{mM}$ for $1 \mathrm{~h}$. The reduced sample was then desalted using gravity columns Nap10 (GE Healthcare Life Sciences) in nitrogen bubbled PBS (pH 7.4) and immediately added to five molar excess of maleimidefunctionalized Alexa647 (Thermo Scientific) for $1 \mathrm{~h}$. Excess of free dye was separated from the conjugated nanobody with an Äkta HPLC equipped with a Superdex 75 increase column (GE Healthcare Life Sciences).

Generation of hoSyn fibrils. Monomeric haSyn was expressed recombinantly in E. coli BL21 (DE3) and purified by anion exchange and size-exclusion chromatography ${ }^{58}$. Cell lysis was conducted by French press (Avestin EmulsiFlex-C3) with lysis buffer (10 mM Tris-HCl, pH 8, $1 \mathrm{mM}$ EDTA, $1 \mathrm{mM}$ PMSF), $20 \mathrm{~mL}$ per $1 \mathrm{~L}$ of cell culture. The lysate was then heated up to $96{ }^{\circ} \mathrm{C}$ for $30 \mathrm{~min}$ in a water bath and centrifuged afterwards for $30 \mathrm{~min}$ at $4{ }^{\circ} \mathrm{C}$ with $22,000 \times g$ (Beckman Coulter, JA 25-5 rotor). Streptomycin was added at a final concentration of $10 \mathrm{mg} / \mathrm{mL}$ and incubated for $15 \mathrm{~min}$. Following another centrifugation step, the soluble protein was precipitated by ammonium sulfate for $15 \mathrm{~min}$ at $4{ }^{\circ} \mathrm{C}$. Protein pellet was dialyzed overnight against $25 \mathrm{mM}$ Tris- $\mathrm{HCl}, \mathrm{pH} 7.7$ and loaded on an anion exchange column (GE Healthcare, Mono Q 5/50 GL). Protein was eluted with $300 \mathrm{mM} \mathrm{NaCl}$. Monomeric haSyn purity and was achieved by size-exclusion chromatography (GE Healthcare, Superdex 75 10/300 GL) using 50 mM HEPES, $\mathrm{pH} 7.4,100 \mathrm{mM} \mathrm{NaCl}$, and $0.02 \% \mathrm{NaN}_{3}$. The protein was sterile filtered $(0.22-\mu \mathrm{m})$ and stored at $1 \mathrm{mM}$ at $-80{ }^{\circ} \mathrm{C}$. Monomeric haSyn in $50 \mathrm{mM}$ HEPES, pH 7.4, $100 \mathrm{mM} \mathrm{NaCl}$, and $0.02 \% \mathrm{NaN}_{3}$ was centrifuged at $84,000 \times \mathrm{g}$ for $1 \mathrm{~h}$ at $4{ }^{\circ} \mathrm{C}$. The supernatant was filtrated through $0.22 \mu \mathrm{m}$ ULTRAFREE-MC centrifugal filter units (Millipore) and adjusted to $0.25 \mathrm{mM}$ protein concentration. Aggregation was performed for 10 days at $37^{\circ} \mathrm{C}$ with constant stirring at $200 \mathrm{rpm}$. Progress of fibril formation was monitored with a Thioflavin $\mathrm{T}$ fluorescence assay ${ }^{76}$. The fibrils were finally collected by ultracentrifugation at $20^{\circ} \mathrm{C}$, washed twice with $50 \mathrm{mM}$ HEPES, $\mathrm{pH} 7.4,100 \mathrm{mM} \mathrm{NaCl}$ and quantified by subtracting the amount of monomeric haSyn in the supernatant from the total protein used for aggregation. Prior to their use, fibrils were resuspended in the washing buffer at $0.1 \mathrm{mM}$ protein concentration.

Proteasome inhibition. MG132 (Sigma-Aldrich, St. Louis, MO, USA) was administrated at different concentrations (Supplementary Fig. 2) or at $1 \mu \mathrm{M}$ to the culture medium for different time intervals (Fig. 2).

CSF samples. Study participants consisted of individuals who were in treatment at the Paracelsus Elena Klinik, Kassel, Germany, and had been diagnosed with a 
variety of neurological disorders non-related to aSyn aggregation disorders. The study cohort consisted of 23 females and 19 male individuals with a mean age of $70.95 \pm 1.51$. For a detailed presentation of demographic and clinical features of participants please see Supplementary Table 1. CSF samples from all individuals were collected after the informed consent of the participant at the Paracelsus Elena Klinik in accordance with the principles of Declaration of Helsinki and following identical standard operating procedures. In brief, CSF was collected by lumbar puncture in the morning with fasting patients in a sitting position. The samples were centrifuged at $2000 \times g$ for $10 \mathrm{~min}$ at room temperature (RT). The concentrations of aSyn in CSF samples were measured with a validated sandwich ELISA system (mSA1/Syn1-BB; 384 well plate format) ${ }^{72}$. Aliquots of the supernatants were frozen within $20-30 \mathrm{~min}$ and stored at $-80^{\circ} \mathrm{C}$ until their use. A total cell count was established in tube $1(2 \mathrm{~mL})$. Samples with erythrocyte counts $>50$ cells per $\mu \mathrm{L}$ CSF in tube 1 were excluded from all analyses. The use of the CSF samples in this study was approved by the ethical committee of the Medical Center Göttingen with the approval numbers 36/7/02 and 9/7/04.

Cell lines. Wild-type HEK293 were grown in Dulbecco's modified Eagle's medium (DMEM) supplemented with 10\% FBS, $4 \mathrm{mM}$ L-glutamine and $600 \mathrm{U} / \mathrm{ml}$ penicillinstreptomycin (Lonza). Reporter-cells (HEK293 stably expressing TetON-NbSyn87EGFP-P2A-mCherry) and haSyn cells (HEK293 stably expressing TetON-haSyn) were grown in DMEM supplemented with $10 \%$ FBS, $2 \mathrm{mM}$ L-glutamine supplemented with $0.5 \mu \mathrm{g} / \mathrm{ml}$ puromycin (InvivoGen, San Diego, CA, USA). Both, Reporter-cells and haSyn cells were produced by Sirion Biotech GmbH (Martinsried, Germany). TetON Induction was performed using $0.5 \mu \mathrm{g} / \mathrm{ml}$ doxycycline (Sigma-Aldrich, St. Louis, MO, USA) at least $12 \mathrm{~h}$ before their use.

Primary hippocampal neurons. Postnatal (P1-P2) pups from Wistar rats were decapitated and the brains were extracted. The hippocampi were isolated, washed in Hank's balanced salt solution (HBSS; Invitrogen, Darmstadt, Germany) and incubated in enzymatic digestion solution for $1 \mathrm{~h}$ at room temperature. After washing in HBSS the hippocampi were incubated in inactivation solution for $15 \mathrm{~min}$. After another washing step in Neurobasal A, neurons were mechanically dissociated by pipetting. In all, 15,000 neurons per well were added to the plating medium (MEM, $10 \%$ horse serum, $3.3 \mathrm{mM}$ glucose, $2 \mathrm{mM}$ glutamine) in PLL-coated 96-multiple glass bottom well plates (SensoPlate, Greiner Bio-One International GmbH, Kremsmünster, Austria) and kept at $37^{\circ} \mathrm{C}, 5 \% \mathrm{CO}_{2}$. After $\sim 1 \mathrm{~h}$, when the neurons adhered to the glass bottom, the plating medium was exchanged with $100 \mu \mathrm{L}$ Neurobasal A and plates were further cultured at $37^{\circ} \mathrm{C}, 5 \% \mathrm{CO}_{2}$. To maintain healthy cultures, $50 \mu \mathrm{L}$ of medium was removed every second day and replaced with $50 \mu \mathrm{L}$ of fresh Neurobasal A. The primary rat hippocampal neuron cultures were prepared with minor modifications from the original protocol ${ }^{77}$. Five days after plating, neurons were infected with an AAV (88e5 TU AAV/5 $\mu$ l) containing the sequence for NbSyn87 fused to mCherry and NLS sequences (NbSyn87-mCherry-NLS).

\section{Cellular uptake of recombinant hoSyn from the culture medium. Purified} synuclein proteins were introduced to the medium of (i) neurons cultured on 96-well plates 6 days post infection with AAV NbSyn87-mCherry-NLS and (ii) Reporter-cells cultured on 24 -well plates $48 \mathrm{~h}$ ( 70,000 cells per well) post-seeding. haSyn (monomers or fibrils) and $\mathrm{h} \beta$ Syn proteins were diluted in Opti-MEM and incubated with Lipofectamine RNAimax (Invitrogen, Thermo Fisher Scientific, 2 and $0.3 \mu \mathrm{l}$ per well for Reporter-cells and neurons, respectively) for $20 \mathrm{~min}$ and then administered to the culture medium at a final concentration of $20 \mu \mathrm{M}$ protein per well. Cells were fixed $14 \mathrm{~h}$ later for imaging and evaluation.

Detection of hoSyn species in CSF samples. Wild-type HEK293 and Reportercells were seeded $(\sim 14,000$ per well) and induced in a PLL-coated 96-well plate with glass bottom (SensoPlate, Greiner Bio-One International GmbH, Kremsmünster, Austria). After $24 \mathrm{~h}$ of induction, the cells were exposed to CSF samples. In brief, the medium was partially eliminated leaving $25 \mu \mathrm{l}$ per well, and supplemented with $50 \mu \mathrm{l}$ of CSF or culture medium as negative control. Cells were further incubated for $24 \mathrm{~h}$ and then fixed by adding $4 \%$ paraformaldehyde in PBS $(137 \mathrm{mM} \mathrm{NaCl}, 2.7 \mathrm{mM}$ $\mathrm{KCl}, 10 \mathrm{mM} \mathrm{Na} 2 \mathrm{HPO}_{4}, 2 \mathrm{mM} \mathrm{KH}_{2} \mathrm{PO}_{4} ; \mathrm{pH}$ 7.4) overnight at $4{ }^{\circ} \mathrm{C}$, aldehyde groups were quenched with $0.1 \mathrm{M} \mathrm{NH}_{4} \mathrm{Cl}$ for $15 \mathrm{~min}$ and images were acquired.

Immunostaining. Cells were briefly washed with DPBS (Reporter-cells) or Tyrode buffer (primary rat hippocampal neurons), fixed with $4 \%$ paraformaldehyde in PBS for $30 \mathrm{~min}$ at RT. Remaining reactive aldehyde groups were quenched in PBS supplemented with $0.1 \mathrm{M}$ glycine and $0.1 \mathrm{M} \mathrm{NH}_{4} \mathrm{Cl}$ for $15 \mathrm{~min}$ at RT. Cells were permeabilized and unspecific protein binding sites were blocked with a blocking/ permeabilization solution $(0.1 \%$ Triton X-100 and $2 \%$ bovine serum albumin, BSA in PBS) at RT for $15 \mathrm{~min}$. Reporter-cells were incubated with a rabbit polyclonal anti- $\alpha / \beta$-Synuclein antibody (dilution 1:500; Cat. No. 128002, SySy, Göttingen, Germany) and neurons with a polyclonal guinea pig anti-synaptophysin antibody (dilution 1:100 Cat. No. 101004, SySy, Göttingen, Germany) for $1 \mathrm{~h}$. After three thorough washing steps, cells were incubated with the secondary antibodies (donkey anti-rabbit, dilution 1:500; Cat. No. 711-175-152, Dianova, Hamburg, Germany or donkey anti-guinea pig IgG labeled with ATTO 647 N, dilution 1:500; Cat. No. N0602-At647N-S, SySy, Göttingen, Germany) for $45 \mathrm{~min}$ or $1 \mathrm{~h}$, respectively. The samples were again subjected to three thorough washing steps with PBS and high-salt PBS $\left(500 \mathrm{mM} \mathrm{NaCl}, 2.7 \mathrm{mM} \mathrm{KCl}, 10 \mathrm{mM} \mathrm{Na}_{2} \mathrm{PO}_{4}, 2 \mathrm{mM}\right.$ $\mathrm{KH}_{2} \mathrm{PO}_{4} ; \mathrm{pH}$ 7.3-7.4). Before mounting the coverslips or before imaging, cells were stained with Höchst $33342(1 \mu \mathrm{g} / \mathrm{ml}$; Thermo Fisher Scientific). Coverslips were finally mounted in Mowiol mounting media $(6 \mathrm{~g}$ glycerol, $6 \mathrm{ml}$ deionized water, $12 \mathrm{ml} 0.2 \mathrm{M}$ Tris buffer $\mathrm{pH}$ 8.5, $2.4 \mathrm{~g}$ Mowiol 4-88 from Merck). In all, 96-well plates were imaged in PBS.

Plasmid electroporation of Xenopus laevis tadpoles. All procedures for animal handling were approved by the governmental animal care and use office (Niedersächsisches Landesamt für Verbraucherschutz und Lebensmittelsicherheit, Oldenburg, Germany, Az.12/0779) and were in accordance with the German Animal Welfare Act as well as with the guidelines of the Göttingen University Committee for Ethics in Animal Experimentation.

$X$. laevis tadpoles (albinos, stage 53) 78 $^{7}$ were used for the in vivo experiments. Injection micropipettes were pulled from borosilicate microcapillaries (Warner instruments; outer diameter: $1.0 \mathrm{~mm}$, inner diameter: $0.58 \mathrm{~mm}$, length $100 \mathrm{~mm}$ ) using a horizontal puller (P 1000, Sutter Instruments). Micropipette tips were sharpened at an angle of $20-30^{\circ}$ until the pipette tip had a syringe-like shape (Micropipette Beveler 48000; World Precision Instruments). Micropipettes were filled with $3 \mu \mathrm{l}$ of plasmid solution/s ( $600 \mathrm{ng} / \mu \mathrm{l})$. Cascade blue dextran $(3 \mathrm{mM}, 10 \%$, Thermo Fisher) was added to the plasmid solution before to be able to observe dye extrusion under fluorescent illumination. Albino tadpoles were anaesthetized in 0.02\% MS-222 (ethyl 3-aminobenzoate methanesulfonate; Sigma-Aldrich; pH: 7.6) for $5 \mathrm{~min}$ until complete immobility and irresponsiveness. Subsequently, the animal was transferred to a moistened dish under a stereomicroscope with brightfield and fluorescent illumination (Olympus SZX16; light source: X-Cite Series $120 \mathrm{Q}$, Lumen dynamics). The glass pipettes filled with plasmid solution/s were mounted to a micromanipulator connected to a Femtojet injection system (Eppendorf). The micropipette was carefully penetrated into the olfactory mucosa at three to five different locations without injuring major arteries. Up to five pressure pulses of $250-1000 \mathrm{hPa}(1 \mathrm{~s}$ each) were applied per site. Once homogenous blue fluorescent signal was visible throughout the olfactory mucosa, an external electric field was applied to the olfactory mucosa using an electroporation setup ${ }^{79}$. One of the $0.2 \mathrm{~mm}$ platinum wire electrodes was positioned in the water-filled nostril, the other one in contact to the skin, laterally to the olfactory nerve. Trains of three square pulses $(20 \mathrm{~V}, 500 \mathrm{~ms}$ duration, and $25 \mathrm{~ms}$ delay) were applied four times in alternating polarity (ELP-01D, NPI Electronics; additional capacitor connected in parallel: Domoport, $3 \mu \mathrm{F})$. The entire procedure was performed in $>5$ min to grant proper anesthesia and avoid dissipation of plasmid solution before electroporation. Following electroporation, animals were put into water until they woke from anesthesia. After assessment of normal swimming the larvae were left in their aquaria to recover for at least $24 \mathrm{~h}$. Individual nostrils were electroporated sequentially, with a $1 \mathrm{~h}$ recovery period in between. We injected and electroporated two plasmids in the left nostril: one expressing FluoReSyn and the other haSyn, both under the CMV promotor. In the right nostril, only the FluoReSyn expressing plasmid was electroporated.

Imaging. Conventional epifluorescence images of the Reporter-cells were obtained with an Olympus IX71 microscope equipped with a $0.5 \mathrm{NA} / \times 20$ dry UPlanFL N objective and captured with an Olympus F-View II CCD camera (Olympus, Hamburg, Germany). Experiments with CSF samples and neurons on 96-well plates were acquired using a Biotek Cytation 3 Imaging Reader (BioTek Instruments, Winooski, VT, USA) equipped with a $\times 20$, Plan Fluorite WD 6.6 NA 0.45 objective, a $465 \mathrm{~nm}$ LED cube (Cat\# 1225001), EGFP Filter cube (Cat\# 1225101), $523 \mathrm{~nm}$ LED cube (Cat\# 1225003), RFP filter cube (Cat\# 1225103) and a 16-bit monochromatic CCD camera (pixel size $6.45 \mu \mathrm{m} \times 6.45 \mu \mathrm{m}$ ).

In vivo multiphoton imaging of the Xenopus olfactory system. For in vivo imaging, we anesthetized the electroporated tadpoles in 0.02\% MS-222 (ethyl 3-aminobenzoate methanesulfonate; Sigma-Aldrich; $\mathrm{pH}$ : 7.6) for $5 \mathrm{~min}$ until complete irresponsiveness. The animals were placed into a recess of a silicone-filled recording chamber. The chamber was filled with water and the animal was mechanically fixed using parafilm. A small window was cut into the parafilm to expose the olfactory organs. Both nostrils were imaged under a two-photon microscope (Nikon A1R MP) at an excitation wavelength of $920 \mathrm{~nm}$. All animals $(n=9)$ and nostrils were imaged as 3D image stacks under the same gain and laser settings, to compare fluorescence intensities of the FluoReSyn. The imaging procedure did not last longer than $10 \mathrm{~min}$ and animals were transferred to a big waterfilled beaker until they recovered from anesthesia.

Cell lysate preparation. Reporter-cells were washed briefly with ice-cold DPBS and lysed with $50 \mu \mathrm{l}$ of lysis buffer per well $(50 \mathrm{mM}$ Tris/ $\mathrm{HCl} \mathrm{pH} 7.5,150 \mathrm{mM}$ $\mathrm{NaCl}, 2 \mathrm{mM}$ EDTA, $0.5 \%$ IgePAL, $0.5 \%$ Sodium deoxycholate and freshly added $250 \mu \mathrm{M}$ PMSF, 10 ng Leupeptin, 10 ng Aprotinin, 1 ng Pepstatin A, 10 ng DNase und $1 \mu \mathrm{l}$ Halt Protease Inhibitor Cocktail; Thermo Fisher Scientific). Cell lysates were collected into a pre-cooled tube and were centrifuged for at least $1 \mathrm{~h}$ at $\sim 15,000 \times g$ at $4{ }^{\circ} \mathrm{C}$. The supernatant was collected into two tubes, snap-frozen with liquid nitrogen and stored at $-80^{\circ} \mathrm{C}$ until needed. 
Western blotting. Reporter-cell lysates were thawed on ice and diluted accordingly to their total protein (determined using BCA assay (Merk)) to load the same total protein concentration in each lane. Samples were mixed with pre-heated $5 \times$ Laemmli buffer (50 mM Tris- $\mathrm{HCl}, 4 \%$ sodium doedecyl sulfate (SDS), $0.01 \%$ Serva Blue G, $12 \%$ glycerol, $\mathrm{pH} 6.8,50 \mathrm{mM}$ DTT) to be further boiled at $95^{\circ} \mathrm{C}$ for $10 \mathrm{~min}$, centrifuged and then loaded into previously casted 10-12\% PAGE. After the SDSPAGE run was completed, proteins were transferred to a nitrocellulose membrane in wet trans blot cell (Biorad) with $400 \mathrm{~mA}$ for $2 \mathrm{~h}$ at $4{ }^{\circ} \mathrm{C}$ while stirring the transfer buffer ( $25 \mathrm{mM}$ Tris, $192 \mathrm{mM}$ glycine, $\mathrm{pH} 8.3$ and $20 \%$ methanol, and $0.04 \%$ SDS). The membrane was incubated for $1 \mathrm{~h}$ in blocking buffer $(5 \%$ Nonfat Dried Milk, $0.1 \%$ Tween 20 in PBS) and then was further incubated with a mouse monoclonal anti-EGFP antibody (1:500; Cat. No. A11120, Invitrogen, Thermo Fisher Scientific) or a rabbit polyclonal anti- $\beta$-Actin-Cy5 antibody (1:1000; Cat. No. 251003, SySy, Göttingen, Germany). Primary antibody incubations were performed overnight at $4{ }^{\circ} \mathrm{C}$ with constant shaking. The following day, the membrane was washed thoroughly in blocking buffer and incubated with the fluorescently labeled secondary donkey polyclonal anti-mouse antibody (1:1000; Cat. No. 715-175-150, Dianova) for $1 \mathrm{~h}$ at RT. For experiments with the directly labeled anti- $\beta$-Actin antibody, the second $1 \mathrm{~h}$ incubation step was omitted. Membrane was washed several times each in $0.1 \%$ Tween 20 in PBS and imaged using an Amersham Imager 600 (GE Healthcare Life Sciences, Little Chalfont, UK).

Dot-Blot. Proteins were serial diluted in washing buffer $(0.05 \%$ Tween 20 in PBS) and spotted on a nitrocellulose membrane. After the membrane was dried, it was blocked with $2 \%$ FBS or BSA, 5\% Nonfat Dried Milk, 0.05\% Tween20 in PBS for $1 \mathrm{~h}$ under agitation. This was followed by incubation with the fluorescently labeled nanobody NbSyn87-Alexa647 for $1 \mathrm{~h}$. Unbound nanobodies were washed away by several thorough washing steps with $0.05 \%$ Tween 20 in PBS for a total duration of $1 \mathrm{~h}$. Finally, images of the membranes were taken with an Amersham Imager 600 (GE Healthcare Life Sciences, Little Chalfont, UK) to detect the NbSyn87-Alexa647 signal.

Data analysis and statistics. Image analyses of experiments presented in Figs. 1, 2, 4-7, and Supplementary Figures were performed with custom-written procedures in Matlab (MathWorks Inc., Natick, MA, USA). Cells were identified automatically based on their Höchst 33342 (Figs. 1, 2, 4-6, Supplementary Fig.) or mCherry (Fig. 7) signals. The average signal intensity within a cell was calculated and corrected for the background intensity by subtracting the background region of interest from the average signal intensity.

Cells were considered as positive if their background-corrected GFP (Figs. 1, 4, 5, Supplementary Fig. 3, 4) or mCherry (Fig. 6) signal intensity (AU) was above the mean plus 2 standard deviations of control GFP or mCherry signal intensity, respectively. For Fig. 7, cells were considered as positive if background-corrected GFP signal intensity was above $400 \mathrm{AU}$. In Fig. 7, signal intensity was normalized to mCherry to exclude differences in FluoReSyn induction.

Graph plotting as well as statistical analyses of data presented in Figs. 1, 2, 4-7, and Supplementary Figures were carried out using custom-written procedures in Matlab, Sigma Plot (Systat Software, San Jose, CA, USA) or GraphPad Prism 5.0 (San Diego, CA, USA). All values are given as mean \pm standard error of the mean from at least three independent experiments. Statistical significance was assessed by one-way ANOVA and Tukey's Post hoc test.

Images showing FluoReSyn signal in vivo (Fig. 3) were created from multichannel 3D image stacks acquired with the multiphoton microscope. Autofluorescence from melanophores was removed by subtracting the maximum intensity z-projections of the blue emission channel (where only autofluorescence was visible) from the green emission channel (with FluoReSyn signal only) using Fiji "Image Calculator" function ${ }^{80}$. For quantitative analysis of differences in FluoReSyn fluorescence between nostrils, maximum intensity z-projections of the green emission channel were processed in Fiji to obtain a binary mask of the regions of interest (nuclei with FluoReSyn signal). Therefore, binary images were created using Li's method of minimum cross-entropy thresholding ${ }^{81}$ followed by four rounds of despeckeling in Fiji ${ }^{80}$. The resulting binary masks were used to measure mean fluorescence intensity values of all regions of interest in the images with areas between 20 and 150 px (size range of nuclei). Fluorescence mean intensities were pooled for each condition (FluorReSyn + haSyn vs. only FluoReSyn) from nine (559 nuclei in total) animals measured under the same conditions. Scatter plots were created using the Seaborn package in Python (python.org; version 0.9; 10.5281/zenodo.1313201).

Reporting summary. Further information on research design is available in the Nature Research Reporting Summary linked to this article.

\section{Data availability}

The data that support the finding of this study is readily available within this paper, its Supplementary file and in the Source Data file. The data set for Figs. 1e, 2b, c, 3i, 4b, $5 b, 6 b, c, 7 b$ and Supplementary Fig. $1 \mathrm{a}-\mathrm{c}, 3 \mathrm{a}, \mathrm{b}, 5 \mathrm{~b}$, and 6 are provided in the Source Data file. All data sets generated during and/or analyzed during the current study are available from the corresponding author on reasonable request.

\section{Code availability}

The customized code generated during and/or analyzed during the current study is available from the corresponding author on reasonable request.

Received: 12 November 2019; Accepted: 5 May 2020;

Published online: 01 June 2020

\section{References}

1. Spillantini, M. G. \& Goedert, M. The a-synucleinopathies: Parkinson's disease, dementia with Lewy bodies, and multiple system atrophy. Ann. NY Acad. Sci. 920, 16-27 (2006)

2. Jakes, R., Spillantini, M. G. \& Goedert, M. Identification of two distinct synucleins from human brain. FEBS Lett. 345, 27-32 (1994).

3. Lautenschläger, J. et al. C-terminal calcium binding of $\alpha$-synuclein modulates synaptic vesicle interaction. Nat. Commun. 9, 712 (2018).

4. Burré, J. et al. Synuclein promotes SNARE-complex assembly in vivo and in vitro. Science 329, 1663-1667 (2010).

5. Rodriguez-Araujo, G. et al. Alpha-synuclein elicits glucose uptake and utilization in adipocytes through the Gab1/PI3K/Akt transduction pathway. Cell Mol. Life Sci. 70, 1123-1133 (2013).

6. Hashimoto, M. et al. alpha-Synuclein protects against oxidative stress via inactivation of the c-Jun N-terminal kinase stress-signaling pathway in neuronal cells. J. Biol. Chem. 277, 11465-11472 (2002).

7. Ahn, M., Kim, S., Kang, M., Ryu, Y. \& Kim, T. D. Chaperone-like activities of alpha-synuclein: alpha-synuclein assists enzyme activities of esterases. Biochem. Biophys. Res. Commun. 346, 1142-1149 (2006).

8. Jin, H. et al. Synuclein negatively regulates protein kinase C $\delta$ expression to suppress apoptosis in dopaminergic neurons by reducing p300 histone acetyltransferase activity. J. Neurosci. 31, 2035-2051 (2011).

9. Burré, J., Sharma, M. \& Südhof, T. C. a-Synuclein assembles into higher-order multimers upon membrane binding to promote SNARE complex formation. Proc. Natl Acad. Sci. USA 111, E4274-E4283 (2014).

10. Karpowicz, R. J., Trojanowski, J. Q. \& Lee, V. M.-Y. Transmission of asynuclein seeds in neurodegenerative disease: recent developments. Lab. Invest. 99, 971-981 (2019).

11. Bartels, T., Choi, J. G. \& Selkoe, D. J. a-Synuclein occurs physiologically as a helically folded tetramer that resists aggregation. Nature 477, 107-110 (2011).

12. Fink, A. L. The aggregation and fibrillation of a-synuclein. Acc. Chem. Res. 39, 628-634 (2006)

13. Opazo, F., Krenz, A., Heermann, S., Schulz, J. B. \& Falkenburger, B. H. Accumulation and clearance of $\alpha$-synuclein aggregates demonstrated by timelapse imaging. J. Neurochem 106, 529-540 (2008).

14. Li, J.-Y. et al. Lewy bodies in grafted neurons in subjects with Parkinson's disease suggest host-to-graft disease propagation. Nat. Med. 14, 501-503 (2008).

15. Desplats, P. et al. Inclusion formation and neuronal cell death through neuron-to-neuron transmission of alpha-synuclein. Proc. Natl Acad. Sci. USA 106, 13010-13015 (2009)

16. Luk, K. C. et al. Pathological $\alpha$-synuclein transmission initiates Parkinson-like neurodegeneration in nontransgenic mice. Science 338, 949-953 (2012).

17. Masuda-Suzukake, M. et al. Prion-like spreading of pathological $\alpha$-synuclein in brain. Brain 136, 1128-1138 (2013).

18. Brundin, P. \& Melki, R. Prying into the prion hypothesis for Parkinson's disease. J. Neurosci. 37, 9808-9818 (2017)

19. Peelaerts, W. et al. $a$-Synuclein strains cause distinct synucleinopathies after local and systemic administration. Nature 522, 340-344 (2015).

20. Grozdanov, V. \& Danzer, K. M. Release and uptake of pathologic alphasynuclein. Cell Tissue Res. 373, 175-182 (2018).

21. Vekrellis, K., Xilouri, M., Emmanouilidou, E., Rideout, H. J. \& Stefanis, L. Pathological roles of $\alpha$-synuclein in neurological disorders. Lancet Neurol. 10, 1015-1025 (2011)

22. Lee, H. J. et al. Assembly-dependent endocytosis and clearance of extracellular a-synuclein. Int. J. Biochem. Cell Biol. 40, 1835-1849 (2008).

23. Freundt, E. C. et al. Neuron-to-neuron transmission of $\alpha$-synuclein fibrils through axonal transport. Ann. Neurol. 72, 517-524 (2012).

24. Klucken, J., Outeiro, T. F., Nguyen, P., McLean, P. J. \& Hyman, B. T. Detection of novel intracellular $\alpha$-synuclein oligomeric species by fluorescence lifetime imaging. FASEB J. 20, 2050-2057 (2006).

25. Hansen, C. et al. $a$-Synuclein propagates from mouse brain to grafted dopaminergic neurons and seeds aggregation in cultured human cells. J. Clin Invest. 121, 715-725 (2011).

26. Hansen, C. et al. A novel $\alpha$-synuclein-GFP mouse model displays progressive motor impairment, olfactory dysfunction and accumulation of $\alpha$-synucleinGFP. Neurobiol. Dis. 56, 145-155 (2013). 
27. Outeiro, T. F. et al. Formation of toxic oligomeric $\alpha$-synuclein species in living cells. PLoS ONE 3, e1867 (2008).

28. Stadler, C. et al. Immunofluorescence and fluorescent-protein tagging show high correlation for protein localization in mammalian cells. Nat. Methods 10, 315-323 (2013).

29. Hughes, L. D., Rawle, R. J. \& Boxer, S. G. Choose your label wisely: watersoluble fluorophores often interact with lipid bilayers. PLoS ONE 9, e87649 (2014).

30. Ugalde, C. L., Lawson, V. A., Finkelstein, D. I. \& Hill, A. F. The role of lipids in a -synuclein misfolding and neurotoxicity. J. Biol. Chem. 294, 9016-9028 (2019).

31. Luk, K. C. et al. Intracerebral inoculation of pathological a-synuclein initiates a rapidly progressive neurodegenerative $\alpha$-synucleinopathy in mice. J. Exp. Med. 209, 975-986 (2012).

32. Rey, N. L., George, S. \& Brundin, P. Spreading the word: Precise animal models and validated methods are vital when evaluating prion-like behaviour of alpha-synuclein. Neuropathol. Appl. Neurobiol. 42, 51-76 (2016).

33. De Genst, E., Messer, A. \& Dobson, C. M. Antibodies and protein misfolding: from structural research tools to therapeutic strategies. Biochim. Biophys. Acta 1844, 1907-1919 (2014).

34. Vaikath, N. N. et al. Antibodies against alpha-synuclein: tools and therapies. J. Neurochem. 150, 612-625 (2019).

35. Muyldermans, S. Nanobodies: natural single-domain antibodies. Annu. Rev. Biochem. 82, 775-797 (2013).

36. Maidorn, M., Olichon, A., Rizzoli, S. O. \& Opazo, F. Nanobodies reveal an extra-synaptic population of SNAP-25 and Syntaxin 1A in hippocampal neurons. $m A$ s s 11, 305-321 (2019).

37. Götzke, H. et al. The ALFA-tag is a highly versatile tool for nanobody-based bioscience applications. Nat. Commun. 10, 4403 (2019).

38. Caussinus, E., Kanca, O. \& Affolter, M. Fluorescent fusion protein knockout mediated by anti-GFP nanobody. Nat. Struct. Mol. Biol. 19, 117-122 (2012).

39. Vuchelen, A. et al. (1) $\mathrm{H},(13) \mathrm{C}$ and (15) $\mathrm{N}$ assignments of a camelid nanobody directed against human alpha-synuclein. Biomol. NMR Assign. 3, 231-233 (2009).

40. Guilliams, T. et al. Nanobodies raised against monomeric $\alpha$-synuclein distinguish between fibrils at different maturation stages. J. Mol. Biol. 425, 2397-2411 (2013).

41. El-Turk, F. et al. Structural effects of two camelid nanobodies directed to distinct C-terminal epitopes on a-synuclein. Biochemistry 55, 3116-3122 (2016).

42. Butler, D. C. et al. Bifunctional anti-non-amyloid component $\alpha$-synuclein nanobodies are protective in situ. PLoS ONE 11, e0165964 (2016).

43. De Genst, E. J. et al. Structure and properties of a complex of $\alpha$-synuclein and a single-domain camelid antibody. J. Mol. Biol. 402, 326-343 (2010).

44. Chatterjee, D. et al. Proteasome-targeted nanobodies alleviate pathology and functional decline in an a-synuclein-based Parkinson's disease model. NPJ Park. Dis. 4, 25 (2018)

45. Haselbach, D. et al. Long-range allosteric regulation of the human $26 \mathrm{~S}$ proteasome by $20 \mathrm{~S}$ proteasome-targeting cancer drugs. Nat. Commun. 8 , 15578 (2017)

46. Hamazaki, J. et al. Rpn10-mediated degradation of ubiquitinated proteins is essential for mouse development. Mol. Cell Biol. 27, 6629-6638 (2007).

47. Kim, J. H. et al. High cleavage efficiency of a $2 \mathrm{~A}$ peptide derived from porcine teschovirus-1 in human cell lines, zebrafish and mice. PLoS ONE 6, e18556 (2011).

48. Blum, M. \& Ott, T. Xenopus: an undervalued model organism to study and model human genetic disease. Cells Tissues Organs 205, 303-313 (2019).

49. Haas, K., Jensen, K., Sin, W. C., Foa, L. \& Cline, H. T. Targeted electroporation in Xenopus tadpoles in vivo -from single cells to the entire brain. Differentiation 70, 148-154 (2002).

50. Zuris, J. A. et al. Cationic lipid-mediated delivery of proteins enables efficient protein-based genome editing in vitro and in vivo. Nat. Biotechnol. 33, 73-80 (2015).

51. Luk, K. C. et al. Exogenous alpha-synuclein fibrils seed the formation of Lewy body-like intracellular inclusions in cultured cells. Proc. Natl Acad. Sci. USA 106, 20051-20056 (2009).

52. Karpinar, D. P. et al. Pre-fibrillar $\alpha$-synuclein variants with impaired Bstructure increase neurotoxicity in parkinson's disease models. EMBO J. 28, 3256-3268 (2009).

53. Delenclos, M. et al. Investigation of endocytic pathways for the internalization of exosome-associated oligomeric alpha-synuclein. Front. Neurosci. 11, 172 (2017).

54. Nonaka, T., Watanabe, S. T., Iwatsubo, T. \& Hasegawa, M. Seeded aggregation and toxicity of $a$-synuclein and tau: cellular models of neurodegenerative diseases. J. Biol. Chem. 285, 34885-34898 (2010).

55. Kim, H. Y. et al. Structural properties of pore-forming oligomers of $\alpha-$ synuclein. J. Am. Chem. Soc. 131, 17482-17489 (2009).
56. Volpicelli-Daley, L. A., Luk, K. C. \& Lee, V. M. Y. Addition of exogenous asynuclein preformed fibrils to primary neuronal cultures to seed recruitment of endogenous $\alpha$-synuclein to Lewy body and Lewy neurite-like aggregates. Nat. Protoc. 9, 2135-2146 (2014).

57. Karpowicz, R. J. et al. Selective imaging of internalized proteopathic -synuclein seeds in primary neurons reveals mechanistic insight into transmission of synucleinopathies. J. Biol. Chem. 292, 13482-13497 (2017).

58. Hoyer, W. et al. Dependence of $\alpha$-synuclein aggregate morphology on solution conditions. J. Mol. Biol. 322, 383-393 (2002).

59. Bousset, L. et al. Structural and functional characterization of two alphasynuclein strains. Nat. Commun. 4, 2575 (2013).

60. Holmes, B. B. et al. Heparan sulfate proteoglycans mediate internalization and propagation of specific proteopathic seeds. Proc. Natl Acad. Sci. USA 110 E3138-E3147 (2013).

61. Brahic, M., Bousset, L., Bieri, G., Melki, R. \& Gitler, A. D. Axonal transport and secretion of fibrillar forms of $\alpha$-synuclein, $A \beta 42$ peptide and HTTExon 1. Acta Neuropathol. 131, 539-548 (2016).

62. Rizzo, G. et al. Accuracy of clinical diagnosis of dementia with Lewy bodies: a systematic review and meta-analysis. J. Neurol. Neurosurg. Psychiatry $\mathbf{8 9}$, 358-366 (2018).

63. Rizzo, G. et al. Accuracy of clinical diagnosis of Parkinson disease: a systematic review and meta-analysis. Neurology 86, 566-576 (2016).

64. Jellinger, K. A. Accuracy of clinical diagnosis of Parkinson disease: a systematic review and meta-analysis. Neurology 87, 237-238 (2016).

65. Majbour, N. K. et al. Longitudinal changes in CSF alpha-synuclein species reflect Parkinson's disease progression. Mov. Disord. 31, 1535-1542 (2016).

66. Stuendl, A. et al. Induction of $a$-synuclein aggregate formation by CSF exosomes from patients with Parkinson's disease and dementia with Lewy bodies. Brain 139, 481-494 (2016).

67. Shi, M. et al. Plasma exosomal $\alpha$-synuclein is likely CNS-derived and increased in Parkinson's disease. Acta Neuropathol. 128, 639-650 (2014).

68. Tsigelny, I. F. et al. Role of $\alpha$-synuclein penetration into the membrane in the mechanisms of oligomer pore formation. FEBS J. 279, 1000-1013 (2012).

69. Volles, M. J. \& Lansbury, P. T. Vesicle permeabilization by protofibrillar $\alpha-$ synuclein is sensitive to Parkinson's disease-linked mutations and occurs by a pore-like mechanism. Biochemistry 41, 4595-4602 (2002).

70. Lashuel, H. A., Hartley, D., Petre, B. M., Walz, T. \& Lansbury, P. T. Neurodegenerative disease: amyloid pores from pathogenic mutations. Nature 418, 291 (2002).

71. Stöckl, M., Claessens, M. M. A. E. \& Subramaniam, V. Kinetic measurements give new insights into lipid membrane permeabilization by $\alpha$-synuclein oligomers. Mol. Biosyst. 8, 338-345 (2012).

72. Mollenhauer, B. et al. $\alpha$-Synuclein and tau concentrations in cerebrospinal fluid of patients presenting with parkinsonism: a cohort study. Lancet Neurol. 10, 230-240 (2011).

73. Maass, F., Schulz, I., Lingor, P., Mollenhauer, B. \& Bähr, M. Cerebrospinal fluid biomarker for Parkinson's disease: an overview. Mol. Cell Neurosci. 97, 60-66 (2019).

74. Parnetti, L. et al. CSF and blood biomarkers for Parkinson's disease. Lancet Neurol. 18, 573-586 (2019).

75. Tang, J. C. Y. et al. Detection and manipulation of live antigen-expressing cells using conditionally stable nanobodies. eLife 5, e15312 (2016).

76. Hoyer, W., Cherny, D., Subramaniam, V. \& Jovin, T. M. Impact of the acidic C-terminal region comprising amino acids $109-140$ on $\alpha$-synuclein aggregation in vitro. Biochemistry 43, 16233-16242 (2004).

77. Kaech, S. \& Banker, G. Culturing hippocampal neurons. Nat. Protoc. 1, 2406-2415 (2006).

78. Nieuwkoop P. \& Faber, J. Normal Table of Xenopus Laevis (Daudin) (Garland Science, 1994).

79. Weiss, L., Offner, T., Hassenklöver, T. \& Manzini, I. Dye electroporation and imaging of calcium signaling in Xenopus nervous system. Methods Mol. Biol. 217-231, 2018 (1865).

80. Schindelin, J. et al. Fiji: an open-source platform for biological-image analysis. Nat. Methods 9, 676-682 (2012).

81. Li, C. \& Lee, C. Minimum cross entropy thresholding. Pattern Recognit. 26, 617-625 (1993).

\section{Acknowledgements}

F.O., F.B.B., I.M., and T.O. were supported by the Deutsche Forschungsgemeinschaft (DFG) through Cluster of Excellence Nanoscale Microscopy and Molecular Physiology of the Brain (CNMPB). We thank Dr. Sebastian Kügler for providing us the AAVs and helpful discussions. We thank Karin Giller, Melanie Wegstroth, Christina Schäfer, and Nicole Hartelt for excellent technical help. Supported by the DFG under Germany's Excellence Strategy - EXC 2067/1- 390729940. Supported in part by the DFG through grant SFB1286/Z3 to S.O.R. 


\section{Author contributions}

C.G., T.O., and E.F.F. designed and conducted experiments, analyzed and interpreted data, and contributed with the writing of the manuscript. N. Waal and H.V. designed and performed experiments, analyzed and interpreted data. N. Wender initially characterized the sensor, designed the Reporter-cell lines, designed and performed experiments, analyzed and interpreted data. I.M. contributed to the interpretation of data and scientific discussions. C.T. and B.M. provided the human CSF samples. T.S., M.Z., and S.B. prepared and provided aSyn fibrils. S.O.R. analyzed and interpreted the data, contributed to the supervision of the study and scientific discussions. F.B.B. designed and performed experiments, analyzed and interpreted the data, supervised the study and wrote the manuscript. F.O. conceived the project, designed and performed experiments, analyzed and interpreted the data, supervised the study and wrote the manuscript.

\section{Competing interests}

F.O. and S.O.R. are shareholders of NanoTag Biotechnologies GmbH. All other authors declare no competing interests.

\section{Additional information}

Supplementary information is available for this paper at https://doi.org/10.1038/s41467020-16575-0.

Correspondence and requests for materials should be addressed to F.O.
Peer review information Nature Communications thanks Alice Chen-Plotkin, Anne Messer and Serge Muyldermans for their contribution to the peer review of this work. Peer reviewer reports are available

Reprints and permission information is available at http://www.nature.com/reprints

Publisher's note Springer Nature remains neutral with regard to jurisdictional claims in published maps and institutional affiliations.

cc) (i) Open Access This article is licensed under a Creative Commons Attribution 4.0 International License, which permits use, sharing, adaptation, distribution and reproduction in any medium or format, as long as you give appropriate credit to the original author(s) and the source, provide a link to the Creative Commons license, and indicate if changes were made. The images or other third party material in this article are included in the article's Creative Commons license, unless indicated otherwise in a credit line to the material. If material is not included in the article's Creative Commons license and your intended use is not permitted by statutory regulation or exceeds the permitted use, you will need to obtain permission directly from the copyright holder. To view a copy of this license, visit http://creativecommons.org/ licenses/by/4.0/.

(C) The Author(s) 2020 